\title{
PROPAGATION AND TIME REVERSAL OF WAVE BEAMS IN ATMOSPHERIC TURBULENCE*
}

\author{
ALBERT C. FANNJIANG ${ }^{\dagger}$ AND KNUT SOLNA AN $^{\ddagger}$
}

\begin{abstract}
We analyze wave propagation in turbulent media using the Gaussian white-noise approximation. We consider two rigorous Gaussian white-noise models: one for the wave field and the other for the Wigner distribution associated with the wave field. Using the white-noise model for the Wigner distribution we show that the interaction of a wave field with the turbulent medium can be characterized in terms of the turbulence-induced entrance aperture. This aperture is proportional to the turbulence-induced coherence length and inversely proportional to the turbulence-induced spread of the wave energy in the transverse wavevectors. The effect of the turbulent medium is important when the turbulence-induced entrance aperture is smaller than the actual entrance aperture. We also study time reversal of the wave field in a turbulent medium and introduce the notion of a turbulence-induced time-reversal aperture which we show is proportional to the turbulence-induced spread in the transmitted wave energy. When the effect of the turbulent medium is important, the turbulence-induced time-reversal aperture corresponds to a time-reversal resolution much better than the resolution in the absence of the turbulent medium. The propagation and spreading of a wave field can be related to time reversal and refocusing of the wave field by a general duality relation, and we present this duality in terms of the uncertainty principle.
\end{abstract}

Key words. multiscale, superresolution, time reversal, turbulence, white-noise approximation, Wigner distribution

AMS subject classifications. 35Q60, 37H10, 34E10, 60F05, 60J65, 78-02

1. Introduction. Waves propagating through atmospheric turbulence are important in a range of applications of increasing importance. Improvements of antenna and laser design with smaller antennas and narrow beam optical-based waves call for a better understanding of how randomness and turbulence affect the propagating wave field. Increased demand for capacity of communication links and enhanced resolution of imaging procedures reinforce this need. Examples of situations when understanding wave turbulence interaction is important are the following:

- Laser communication channels. High-data-rate communication channels between satellites, ground, and aircrafts have become possible. The satelliteto-ground links are particularly sensitive to turbulence in the atmospheric boundary layer.

- Remote sensing, imaging, and target identification. Laser optics technology can be used for weather or pollution prediction as well as various other imaging tasks. Backscattered or transmitted wave fields from natural or active sources and information about how they are affected by the atmosphere can be used to predict, for instance, temperature or the presence of pollutants and turbulence [9].

- Aerial and astronomical imaging. For earthbound observatories, the largest aperture not affected by atmospheric turbulence typically ranges between

* Received by the editors November 3, 2003; accepted for publication (in revised form) May 11, 2004; published electronically March 3, 2005.

http://www.siam.org/journals/mms/3-3/60159.html

${ }^{\dagger}$ Department of Mathematics, University of California at Davis, Davis, CA 95616 (fannjian@math. ucdavis.edu). This author's research was supported by the Centennial Fellowship of the American Mathematical Society, the UC Davis Chancellor's Fellowship, and NSF grant DMS-0306659.

${ }_{\ddagger}$ Department of Mathematics, University of California at Irvine, Irvine, CA 92697 (ksolna@math. uci.edu). This author's research was supported by NSF grant DMS-0307011 and the Sloan Foundation. 
5 and $20 \mathrm{~cm}$ at visible wavelengths with an average value of $10 \mathrm{~cm}$. For larger apertures, the speckle pattern of the star image varies quickly with time, causing twinkling of the star due to the refractive index turbulence. At longer exposure times the patterns add up to a bright extended spot and restrict the angular resolution of large, ground-based telescopes to the "seeing limit" of about 0.5 arc-sec, which is typically far larger than the theoretical diffraction limit (see section 4.3 for a discussion of image resolution in turbulent media). In order to reconstruct diffraction-limited object images, understanding the point-spread function of the combined system of atmosphere and telescope is crucial $[24,25,42]$.

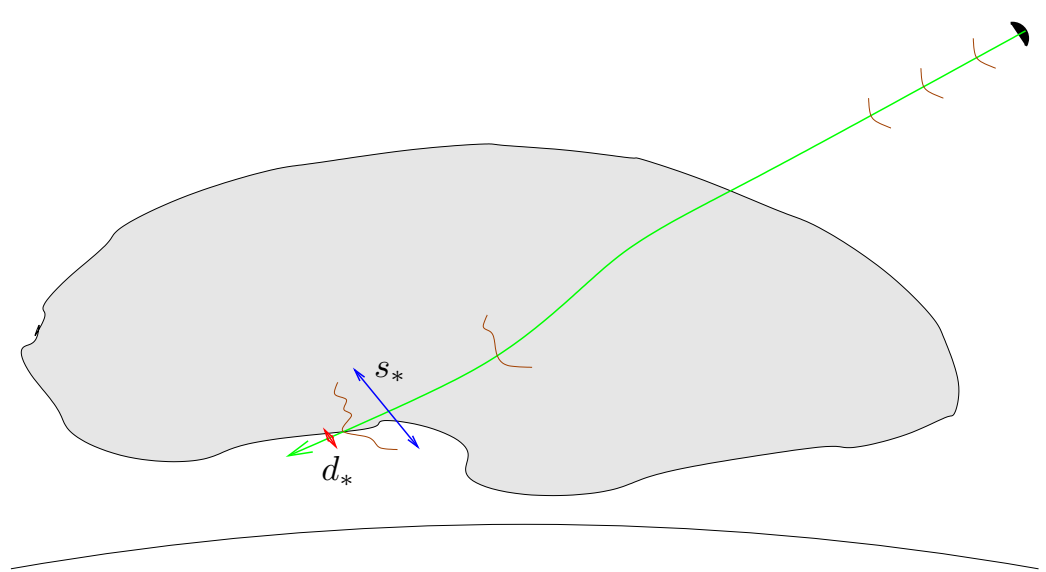

FIG. 1.1. Signals propagating through the atmosphere may be corrupted by atmospheric turbulence. A good model of the propagation process is important in order to be able to design schemes to compensate for this corruption. The wave beam is subject to beam spreading (which we will describe by the spread parameter $s_{*}$ ); moreover, it is subject to displacement and wavefront distortion (which we will describe by the coherence length $d_{*}$ ).

Both for communication and imaging purposes, optical propagation, especially with a coherent source such as laser, is becoming increasingly important. Laser radars with waves in the regime from the ultraviolet to the infrared part of the electromagnetic spectrum have a potential for high capacity and resolution but are, however, more susceptible to the atmospheric effects than the microwave-based radars.

Electromagnetic waves propagating through the atmosphere are subject to attenuation and wavefront distortion (Figure 1.1). Frequency-dependent interaction caused by absorption or scattering can significantly corrupt the signal. The atmosphere extends to approximately $700 \mathrm{~km}$, comprising the atmospheric boundary layer, the troposphere, and stratosphere, with the boundary layer extending to about $2 \mathrm{~km}$, the troposphere to about $20 \mathrm{~km}$, and the stratosphere to about $40 \mathrm{~km}$. Atmospheric conditions that affect the wave can roughly be divided into clear air, clouds, or rain. Here we will be concerned with the more benign clear air situation whose main effect is wavefront distortion or refraction with a negligible degree of attenuation. For optical waves the physical mechanism of refraction is mainly through the small-scale temperature variations which are caused by turbulent eddies and result in the turbulent field of refractive index acting like optical lenses, sometimes referred to as the refractive turbulence. A distinguishing feature of the refractive turbulence is the presence of a wide band of scales causing the refractive turbulence field to be spatially rough or irregular, and this has important consequences in optical propagation. 
In this paper we present a framework for analyzing wave-turbulence interaction and discuss applications to time reversal of waves and scintillation of the wave field. In section 2 we discuss issues related to scales and turbulence modeling. In section 3 we introduce the paraxial wave approximation, its white-noise limit, and the associated moment equations. We give the details of the white-noise limit in various scaling regimes in the appendices. In sections 4 and 5 we discuss the coherent wave, the mutual coherence function, and time reversal of wave packets. In section 6 we derive the duality relation between the forward propagation and its time-reversal counterpart.

2. Length scales. A distinguishing feature of the problems described above is the presence of many length scales. In this section we discuss turbulence parameters, the underlying parameters that characterize the particular physical environment, based on the Kolmogorov theory of turbulence and its variants.

The turbulent atmosphere is very complicated and can be described only in a statistical manner. The Kolmogorov scaling theory plays a crucial role in the statistical modeling of the turbulent atmosphere and is a phenomenological description of the velocity field in the atmosphere. Based on a scaling argument, the mean-square velocity differences are described in a universal manner over a broad range of spatial scales, the inertial range $\left(\ell_{0}, L_{0}\right)$. If we assume homogeneity, isotropy, and incompressibility, the result is that the ensemble average of the squared velocity differences, the so-called structure function, has the form

$$
\mathbb{E}\left[\left(v_{\overrightarrow{\mathbf{x}}}\left(\overrightarrow{\mathbf{x}}_{0}+\overrightarrow{\mathbf{x}}\right)-v_{\overrightarrow{\mathbf{x}}}\left(\overrightarrow{\mathbf{x}}_{0}\right)\right)^{2}\right]=C_{v}^{2}|\overrightarrow{\mathbf{x}}|^{2 / 3}, \quad \overrightarrow{\mathbf{x}} \in \mathbb{R}^{3}, \quad|\overrightarrow{\mathbf{x}}| \in\left(\ell_{0}, L_{0}\right),
$$

where $v_{\overrightarrow{\mathrm{x}}}$ is the velocity in the direction of the displacement $\mathbf{x}$ and $C_{v}$ is the structure parameter of the velocity field. Here and below, $\mathbb{E}$ denotes the ensemble average.

The influence of atmospheric turbulence on the refractive index fluctuation $\delta n$ is through the inertial-convective scale fluctuation $\delta T$ of the temperature field via the relation

$$
\delta n \approx-79 P \frac{\delta \Theta}{T^{2}} \times 10^{-6},
$$

where $P$ is the pressure in millibars, $T$ the temperature in degrees Kelvin, and $\Theta=T-\gamma_{a} h$ the so-called potential temperature with $\gamma_{a}=9.8^{\circ} \mathrm{C} / \mathrm{km}$ and $h$ being the height above ground [38]. The inertial-convective range temperature fluctuation $\delta T$ is usually modeled as a passive scalar field diffusing in and advected by the turbulent velocity field and has a spatial structure function with the same Kolmogorov 2/3 scaling so that

$$
D_{n}(|\overrightarrow{\mathbf{x}}|)=\mathbb{E}[\delta n(\overrightarrow{\mathbf{x}}+\cdot)-\delta n(\cdot)]^{2}=C_{n}^{2}|\overrightarrow{\mathbf{x}}|^{2 / 3}, \quad|\overrightarrow{\mathbf{x}}| \in\left(\ell_{0}, L_{0}\right),
$$

with $\left(\ell_{0}, L_{0}\right)$ the inertial-convective range [34] (see also [12] for rigorous analysis of the evolution of the passive scalar field). The Kolmogorov model for refractive index fluctuations is widely used in order to understand wave propagation in atmospheric turbulence $[3,38]$.

The inertial-convective range of the refractive index fluctuation is bounded by two parameters: the inner scale $\ell_{0}$ and the outer scale $L_{0}$. The inner scale $\ell_{0}$ is typically in the range of $1 \mathrm{~mm}$ to $1 \mathrm{~cm}$, while the outer scale $L_{0}$ is in the range of $100 \mathrm{~m}$ to $1 \mathrm{~km}$. The ratio $L_{0} / \ell_{0}$ grows indefinitely as the Reynolds number of the velocity turbulence increases. Both the inner scale and the outer scale have an impact on wave propagation $[8,17,43]$. Their relative importance depends on the length scales that can be associated with the wave (see below). 
Note that only the instantaneous spatial structure function of the refractive index field is relevant to electromagnetic wave propagation since the period of the oscillation of the wave is typically of the order $10^{-14} \mathrm{~s}$, many orders of magnitude smaller than the time scale of the turbulence.

The refractive index structure function has a spectral representation [38]

$$
D_{n}(|\overrightarrow{\mathbf{x}}|)=8 \pi \int_{0}^{\infty} \Phi_{n}(|\overrightarrow{\mathbf{k}}|)\left[1-\frac{\sin (|\overrightarrow{\mathbf{k}}||\overrightarrow{\mathbf{x}}|)}{|\overrightarrow{\mathbf{k}}||\overrightarrow{\mathbf{x}}|}\right]|\overrightarrow{\mathbf{k}}|^{2} d|\overrightarrow{\mathbf{k}}|
$$

with the power-spectrum density

$$
\Phi_{n}(|\overrightarrow{\mathbf{k}}|)=0.033 C_{n}^{2}|\overrightarrow{\mathbf{k}}|^{-11 / 3}
$$

Here the structure parameter $C_{n}^{2}$ depends in general on the temperature gradient on the large scales $\geq L_{0}$. The latter, in turn, depends on weather conditions, altitude, time of day, season of the year, etc. The typical variation of the structure parameter from ground to $20 \mathrm{~km}$ is about two orders of magnitude in $\mathrm{m}^{-2 / 3}$ according to the data given by [29]. For the case of propagation paths that extend above $1 \mathrm{~km}$ or slant paths that extend from the surface into space, a general model can be formulated which includes a well-known "surge" phenomenon in $C_{n}^{2}$ associated with the turbulent boundary layer near the ground:

$$
C_{n}^{2}(h) \approx C_{0}^{2}\left(h_{2}\right) \exp \left[-h / h_{0}\right]\left[h_{2}^{a} / h^{a}+K \exp \left[-b\left(1-h / h_{1}\right)^{2}\right]\right],
$$

where $h_{1} \sim 1.5-2 \mathrm{~km}, h_{0} \sim 25 \mathrm{~km}$ (i.e., the distance through the troposphere), $C_{0}^{2}\left(h_{2}\right)$ is the value of the structure parameter near the ground at $h_{2} \ll h_{1}$, and $1 / 3<a<4 / 3$. The constant $K<1$ represents the relative strength of the surge layer, and the constant $b$ determines the width of the layer [33]. The scale of variation of $C_{n}^{2}$ is essentially separated from that of the inertial-scale turbulence. Recent analysis of newly acquired data shows that there are substantial variations in the exponent $H$ [37]. In general, the exponents $H$ and $C_{n}^{2}$ vary slowly in space and time and may be regarded as random fields themselves. Important for our approach is that the scales of variation of $H$ and $C_{n}^{2}$ are typically larger than the outer scale of the atmospheric turbulence.

In this paper we consider a general family of power-law type spectra with the spectral exponent $H \in(0,1)$ :

$$
\Phi(\overrightarrow{\mathbf{k}}) \approx 0.033 C_{n}^{2}|\overrightarrow{\mathbf{k}}|^{-1-2 H}|\overrightarrow{\mathbf{k}}|^{-d}, \quad d=2, \quad \text { for }|\overrightarrow{\mathbf{k}}| \in\left(L_{0}^{-1}, \ell_{0}^{-1}\right),
$$

where $d$ is the number of transversal spatial dimensions which we will take to be two. We assume that the spectrum decays sufficiently fast for $|\overrightarrow{\mathbf{k}}| \gg \ell_{0}^{-1}$ while staying bounded for $|\overrightarrow{\mathbf{k}}| \ll L_{0}^{-1}$. In the limit of vanishing inner scale $\ell_{0}=0, H$ is the upper limit of the Hölder regularity of the field and equals $1 / 3$ in the case of the Kolmogorov spectrum. The details of the statistics of the refractive index field are not important for our analysis; only the exponent $H$ is. In general, $H$ may be assumed to vary slowly in space compared to the outer scale of the turbulence. The analysis presented in this paper is capable of treating the case of variable $C_{n}^{2}$ and $H$ as long as their scale of variation is larger than the outer scale. For simplicity of presentation, however, we assume the case with constant $C_{n}^{2}$ and $H$.

Although the self-similar, isotropic spectrum (3.11) is our principal example, the main conclusions (such as superresolution and duality relations) are by no means 
limited to such a medium; they are equally applicable to anisotropic media. However, without loss of generality, we may assume the reflectional symmetry:

$$
\Phi(\overrightarrow{\mathbf{k}})=\Phi(-\overrightarrow{\mathbf{k}}) \quad \forall \overrightarrow{\mathbf{k}} \in \mathbb{R}^{d+1} .
$$

Next, we discuss some important scale parameters that are determined by the specific wave propagation application considered:

- The carrier wavelength $\lambda_{0} \leq 1 \mathrm{~m}$ : Lasers typically generate coherent radiation in the range from the ultraviolet to the infrared band. In the case that the wavelength is below $10^{-1} \mu \mathrm{m}$ (i.e., ultraviolet) the dispersion effect starts to be felt. Our approach is suitable for the microwave range too as long as the width of the wave beam or pulse lies within the inertial-convective range.

- The longitudinal scale $L_{z} \sim 1-20 \mathrm{~km}$ : This is the reference scale in the direction of propagation and should be much larger than the outer scale $L_{0}$ and not too large compared to the scales of variation of $C_{n}^{2}$ and $H$. In astronomical imaging, high-altitude aerial photography, and ground-to-satellite communication links, the wave field will typically propagate through the atmosphere over distances up to $100 L_{0}$.

- The transverse scale $L_{x} \in\left(\ell_{0}, L_{0}\right)$ : This is the reference scale in the lateral direction of the wave beam or pulse. Usually we can set $L_{x}$ to be the width of the wave beam. This reference scale should lie within the inertial-convective scale of the turbulence.

Figure 2.1 summarizes the role of some of the length scales introduced above.

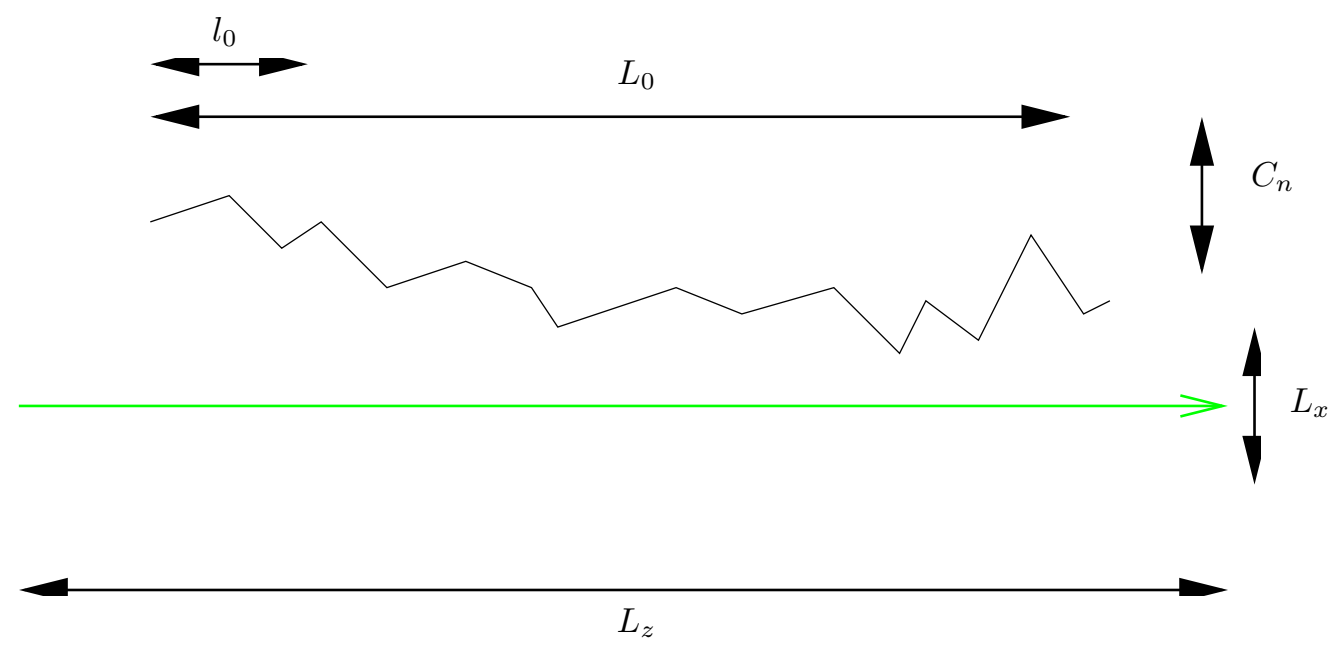

FIG. 2.1. Some important scales present in atmospheric propagation. The inertial subrange is the range in between the inner scale $l_{0}$ and the outer scale $L_{0}$. The strength of the turbulence is measured by $C_{n}$, and $L_{x}, L_{z}$ are typical transversal and longitudinal length scales, respectively.

Though our primary motivation is optical or microwave propagation in the turbulent atmosphere our results are relevant to other situations such as sound wave propagation in the atmosphere, the earth's crust, or biological tissues. Many regions of the earth's crust are very heterogeneous and can be best modeled by incorporating a continuum of scales. Similarly, the interface zone in between different tissue types or the zone associated with damaged tissue might be very rich in scale contents. 
3. Electromagnetic waves in the atmosphere. For optical propagation in the atmosphere, Maxwell's equations simplify considerably. We describe below these simplifications which are used to obtain equations describing the evolution of the moments or multiple correlations of the wave field. Such moment equations are important in the various applications described above.

3.1. Reduced wave equation. We consider time-harmonic waves with $\mathbf{E}$ denoting the electric field. We assume zero conductivity which is appropriate as long as the intensity of the electromagnetic fields is not strong enough to ionize the air molecules. We write the refractive index field $n(\overrightarrow{\mathbf{x}})$ as

$$
n(\overrightarrow{\mathbf{x}})=\bar{n}(1+\tilde{n}(\overrightarrow{\mathbf{x}})),
$$

where $\tilde{n}(\overrightarrow{\mathbf{x}}) \ll 1$ is the relative fluctuation of the refractive index and $\bar{n} \approx 1$ the mean refractive index. The vector wave equation for the electric field is then

$$
\nabla^{2} \mathbf{E}+k^{2}(1+\tilde{n}(\overrightarrow{\mathbf{x}}))^{2} \mathbf{E}+2 \nabla(\mathbf{E} \cdot \nabla \log n)=0,
$$

where $k=\bar{n} \omega / c_{0}$ is the wave number with $c_{0}$ being the speed of light in vacuum. In a small scattering angle regime with $\lambda_{0} \leq$ const. $\ell_{0}$ we can ignore depolarization effects [38] that are due to the third term in (3.1). This corresponds to scatterers being large relative to the wavelength and the direction of the scattered wave very close to the direction of the original scattered wave, which is the case for the regime that we will consider. In this regime the (scalar) Helmholtz equation

$$
\nabla^{2} E+k^{2} E \approx-2 k^{2} \tilde{n} E
$$

with appropriate boundary conditions describes the evolution of the harmonic amplitude for the components of the electric field.

3.2. Parabolic wave equation. For the so-called weak fluctuation regime such as short propagation distances, say less than $100 \mathrm{~m}$, perturbation methods can be used, but such approximations are in general not suited for applications to communication and imaging in the atmosphere. For optical beams in a small cone about the optical axis, the parabolic wave approximation can be used. We assume that the direction of propagation is primarily in the $z$-direction and make the ansatz for the wave equation

$$
E\left(z, \mathbf{x}_{\perp}\right)=\Psi\left(z, \mathbf{x}_{\perp}\right) \exp (i k z), \quad\left(z, \mathbf{x}_{\perp}\right) \in \mathbb{R}^{d+1}, \quad d=2,
$$

where $\mathbf{x}_{\perp} \in \mathbb{R}^{d}$ are the transverse coordinates and $z$ is the longitudinal coordinate. Assuming $\left|\Psi_{z z}\right| \ll 2 k\left|\Psi_{z}\right|$ one obtains the parabolic or paraxial wave equation

$$
i 2 k \frac{\partial \Psi\left(z, \mathbf{x}_{\perp}\right)}{\partial z}+\Delta_{\perp} \Psi\left(z, \mathbf{x}_{\perp}\right)+2 k^{2} \tilde{n}\left(z, \mathbf{x}_{\perp}\right) \Psi\left(z, \mathbf{x}_{\perp}\right)=0, \quad \Psi\left(0, \mathbf{x}_{\perp}\right)=\Psi_{0}\left(\mathbf{x}_{\perp}\right),
$$

where $\Delta_{\perp}$ denotes the Laplacian operator in the transverse coordinates. The physical basis of the parabolic approximation is discussed in $[39,40]$ and derived rigorously in a regime with discretely layered weakly fluctuating media in [1], where the simultaneous limit of parabolic and white-noise approximations is considered. Note that below we drop the subscript indicating the transversal coordinate. 
3.3. White-noise approximation. One cannot solve exactly the parabolic wave equation nor obtain closed-form equations for the moments of the wave field. However, the latter can be achieved by using the white-noise approximation [26] and [40]. In this approximation the wave field becomes a Markov random process whose moments satisfy closed-form equations.

In this section we nondimensionalize coordinates in the parabolic equation (3.2) and discuss a scaling regime where the white-noise approximation can be derived explicitly. Moreover, we discuss the moment equations that follow from this limit. Note that in several wave propagation scenarios it is convenient to work with the Wigner distribution of the wave field rather than the wave field itself, and we shall discuss below the white-noise limit also for the Wigner distribution of the wave field and the associated moment equations.

3.4. Scaling. We introduce dimensionless wave numbers and coordinates by

$$
\tilde{k}=k / k_{0}, \quad \tilde{\mathbf{x}}=\mathbf{x} / L_{x}, \quad \tilde{z}=z / L_{z},
$$

where $k_{0}=2 \pi / \lambda_{0}$ with $\lambda_{0}$ being the characteristic wavelength and $L_{z}$ and $L_{x}$ being the reference scales in the longitudinal and transverse direction, respectively. For example, in view of the separation of scales of $C_{n}^{2}$ and $H$ from those of the inertialconvective range it is natural to use $L_{z} \sim h_{0}$ in (2.4) or the scale of variation in $H$ in the case of vertical propagation through the atmosphere. We are mostly concerned with the situation with $\tilde{k} \approx 1$.

In the new nondimensionalized coordinates the parabolic wave equation becomes

$$
i 2 \tilde{k} \frac{\partial \Psi}{\partial z}+\gamma \Delta \Psi+2 \tilde{k}^{2} k_{0} L_{z} \tilde{n}\left(z L_{z}, \mathbf{x} L_{x}\right) \Psi=0,
$$

after dropping the tilde in the new space coordinates. The Fresnel length $L_{f}$ and Fresnel number $\gamma$ are defined by

$$
\begin{aligned}
L_{f} & =\sqrt{L_{z} \lambda_{0}}, \\
\gamma & =\frac{L_{z}}{L_{x}^{2} k_{0}}=\frac{1}{2 \pi}\left(\frac{L_{f}}{L_{x}}\right)^{2} .
\end{aligned}
$$

The Fresnel length is the diffraction scale which is also the size of the eddies most active in generating wave fluctuations at a distance $L_{z}$ [38]. The relative upper and lower cutoffs of the turbulence spectrum describing the inertial range in the rescaled coordinates are

$$
\eta^{-1}=\frac{L_{0}}{L_{x}}, \quad \rho^{-1}=\frac{\ell_{0}}{L_{x}} .
$$

Essential for the white-noise limit is a small aspect ratio of the wave beam:

$$
\varepsilon^{-2}=\frac{L_{z}}{L_{x}} \gg 1
$$

that is, the wave beam is narrow.

3.5. White-noise scaling. We can now rewrite (3.5) as

$$
i 2 \tilde{k} \frac{\partial \Psi^{\varepsilon}}{\partial z}+\gamma \Delta \Psi^{\varepsilon}+\frac{\tilde{k}^{2}}{\gamma \varepsilon} V\left(\frac{z}{\varepsilon^{2}}, \mathbf{x}\right) \Psi^{\varepsilon}=0 .
$$


The spectrum for the (normalized) process $V$ is given by [15]

$$
\Phi_{(\eta, \rho)}(\overrightarrow{\mathbf{k}}) \approx \sigma_{H}|\overrightarrow{\mathbf{k}}|^{-1-2 H}|\overrightarrow{\mathbf{k}}|^{-d} \quad \text { for }|\overrightarrow{\mathbf{k}}| \in(\eta, \rho)
$$

with

$$
\sigma_{H}=2 \sqrt{0.033} \frac{L_{x}^{H} C_{n}}{\varepsilon^{3}} .
$$

We fix the value $\sigma_{H}$ and aim to analyze (3.10) in the limit $\varepsilon \rightarrow 0$. For a numerical example with $H=1 / 3, L_{z}=10 \mathrm{~km}, L_{x}=1 \mathrm{~m}$, and $C_{n} \sim 10^{-8} \mathrm{~m}^{-1 / 3}$, one has $\sigma_{H} \approx 0.002$ and $\gamma=0.0008$ at the typical optical wavelength of $\lambda_{0}=0.5 \mu \mathrm{m}$. In this example the turbulent medium can have a significant effect on propagation.

3.6. Schrödinger-Itô equation. In this section we introduce the Itô equation describing the Gaussian white-noise limit. We give the precise conditions that allow us to rigorously characterize the solution to the Schrödinger equation in terms of a Schrödinger-Itô equation in the limit when $\varepsilon \rightarrow 0$ in Appendix A.1. Here we describe some main aspects of this limit and the associated moment equations that play a crucial role in applications.

We start by giving the covariance in the lateral spatial variables for the Gaussian white-noise model:

$$
\begin{aligned}
\Gamma^{(\eta, \rho)}(\mathbf{x}-\mathbf{y}) & =\pi \int \cos ((\mathbf{x}-\mathbf{y}) \cdot \mathbf{p}) \Phi_{(\eta, \rho)}(0, \mathbf{p}) d \mathbf{p}, \\
\Gamma_{0}^{(\eta, \rho)} & =\Gamma^{(\eta, \rho)}(0), \quad \eta>0, \quad \rho>0,
\end{aligned}
$$

where we have written the wavevector $\overrightarrow{\mathbf{k}} \in \mathbb{R}^{3}$ as $\overrightarrow{\mathbf{k}}=(\xi, \mathbf{p})$ with $\mathbf{p} \in \mathbb{R}^{2}$. As we describe in Appendix A.1, the $L^{2}$-weak solution $\Psi^{\varepsilon}$ of (3.10) converges in law to the $L^{2}$-weak solution of the Gaussian white-noise model described by the Schrödinger-Itô equation

$$
d \Psi=\left(\frac{i \gamma}{2 \tilde{k}} \Delta-\frac{\tilde{k}^{2}}{4 \gamma^{2}} \Gamma_{0}^{(\eta, \rho)}\right) \Psi d z+\frac{i \tilde{k}}{\gamma \sqrt{2}} d \mathcal{B}_{z} \Psi, \quad \Psi_{0} \in L^{2}\left(\mathbb{R}^{d}\right),
$$

with $\mathcal{B}_{z}$ being a Brownian field with the spatial covariance $\Gamma^{(\eta, \rho)}(\mathbf{x}-\mathbf{y})$ [15]. The limit of vanishing inner scale is now obtained by letting $\rho \rightarrow \infty$ in (3.13). If we also let $\eta \rightarrow 0$, then we need to first subtract a rapidly oscillating and random phase factor in order to arrive at the white-noise limit.

Observe that we started out with medium fluctuations that were isotropic. However, the strongly anisotropic scaling corresponding to a long, narrow beam propagation enabled us to approximate the limiting wave process with a white-noise model which is " $\delta "$-correlated in the $z$-direction but exhibits strong correlation in the lateral directions. The power spectrum of the white-noise model can be obtained from (3.11) and is given by

$$
\Phi_{(\eta, \rho)}(0, \mathbf{p}) \approx \sigma_{H}|\mathbf{p}|^{-1-2(H+1 / 2)}|\mathbf{p}|^{-(d-1)} \quad \text { for }|\mathbf{p}| \in(\eta, \rho), \quad \mathbf{p} \in \mathbb{R}^{d},
$$

giving rise to the modified spectral exponent $H+1 / 2$ in the lateral direction. Thus, long-range dependence in the $z$-direction "spills" over to enhance the lateral persistence in the white-noise model. 
In order to characterize the wave field it is often useful to compute its higher order moments. The $n$-point correlation function for $\Psi$ in the context of the Schrödinger-Itô equation (3.15) is defined by

$$
F^{(n)}\left(z, \mathbf{x}_{1}, \ldots, \mathbf{x}_{n}\right) \equiv \mathbb{E}\left[\Psi\left(z, \mathbf{x}_{1}\right) \cdots \Psi_{z}\left(z, \mathbf{x}_{n}\right)\right],
$$

and it follows from (3.15) that $F^{(n)}$ solves the closed-form equation

$$
\frac{\partial F^{(n)}}{\partial z}=\frac{i \gamma}{2 \tilde{k}} \sum_{j=1}^{n} \Delta_{\mathbf{x}_{j}} F^{(n)}-\frac{\tilde{k}^{2}}{4 \gamma^{2}} \sum_{j, k=1}^{n} \Gamma^{(\eta, \infty)}\left(\mathbf{x}_{j}, \mathbf{x}_{k}\right) F^{(n)} .
$$

As it stands, the moment equations are the Schrödinger equation with inhomogeneous potentials in $n d$ dimensions and thus are not exactly solvable. A more tractable formulation for the moments is through the Wigner distribution. Since the Wigner distribution also appears naturally in the phase-conjugation or time-reversal problems [5] we recall next its white-noise limit.

3.7. Wigner distribution and Wigner-Itô equation. As we will see, the study of phase-conjugated, back-propagated wave fields naturally leads to the Wigner distribution

$$
W^{\varepsilon}(z, \mathbf{x}, \mathbf{p})=\frac{1}{(2 \pi)^{d}} \int e^{-i \mathbf{p} \cdot \mathbf{y}} \Psi^{\varepsilon}\left(z, \mathbf{x}+\frac{\gamma \mathbf{y}}{2}\right) \overline{\Psi^{\varepsilon}\left(z, \mathbf{x}-\frac{\gamma \mathbf{y}}{2}\right)} d \mathbf{y}
$$

with the bar representing complex conjugation. The Wigner distribution is realvalued, and from it one can recover the marginal intensities by partial integration:

$$
\begin{aligned}
\left|\Psi^{\varepsilon}(z, \mathbf{x})\right|^{2} & =\int W^{\varepsilon}(z, \mathbf{x}, \mathbf{p}) d \mathbf{p}, \\
\left|\hat{\Psi}^{\varepsilon}(z, \mathbf{p})\right|^{2} & =\left(\frac{\gamma}{2 \pi}\right)^{d} \int W^{\varepsilon}(z, \mathbf{x}, \gamma \mathbf{p}) d \mathbf{x}
\end{aligned}
$$

so that we may think of $W^{\varepsilon}(\mathbf{x}, \mathbf{p})$ as a wave number-resolved density on the phase space. A closed equation for $W^{\varepsilon}$, called the Wigner-Moyal equation, follows from (3.10) and reads as

$$
\frac{\partial W_{z}^{\varepsilon}}{\partial z}+\frac{\mathbf{p}}{\tilde{k}} \cdot \nabla_{\mathbf{x}} W_{z}^{\varepsilon}+\frac{\tilde{k}}{2 \varepsilon} \mathcal{L} W_{z}^{\varepsilon}=0
$$

where the operator $\mathcal{L}$ defined as

$$
\mathcal{L} W_{z}^{\varepsilon}=i \int e^{i \mathbf{q} \cdot \mathbf{x}} \gamma^{-1}\left[W_{z}^{\varepsilon}(\mathbf{x}, \mathbf{p}+\gamma \mathbf{q} / 2)-W_{z}^{\varepsilon}(\mathbf{x}, \mathbf{p}-\gamma \mathbf{q} / 2)\right] \widehat{V}\left(\frac{z}{\varepsilon^{2}}, d \mathbf{q}\right)
$$

is skew-symmetric and real (i.e., mapping real-valued functions to real-valued functions). As a consequence of the skew symmetry of $\mathbf{p} \cdot \nabla_{\mathbf{x}}$ and $\mathcal{L},(3.21)$ preserves the $L^{2}$-norm of the initial condition. Here $\widehat{V}(z, d \mathbf{q})$ is the (partial) spectral representation for $\nu$,

$$
V(z, \mathbf{x})=\int \exp (i \mathbf{p} \cdot \mathbf{x}) \widehat{V}(z, d \mathbf{p})
$$

where the process $\widehat{V}(z, d \mathbf{p})$ is the (partial) spectral measure of orthogonal increments over $\mathbf{p}$. As in the previous section we will consider the weak formulation of the Wigner equation. 
The precise conditions under which the convergence, as $\varepsilon \rightarrow 0, \rho \rightarrow \infty, \eta \rightarrow 0$, to the white-noise model for the Wigner-Moyal equation (3.21) occurs are given in Appendix A.2. Under the conditions specified in Appendix A the $L^{2}$-weak solution $W^{\varepsilon}$ of the Wigner-Moyal equation with the initial data $W_{0} \in L^{2}\left(\mathbb{R}^{2 d}\right)$ converges in law to the $L^{2}$-weak solution of the Gaussian white-noise model described by the Wigner-Itô equation

$$
d W=\left(\frac{-1}{\tilde{k}} \mathbf{p} \cdot \nabla_{\mathbf{x}}+\frac{\tilde{k}^{2}}{4} \mathcal{Q}_{0}^{(\eta, \rho)}\right) W d z+\frac{\tilde{k}}{\sqrt{2}} d \widetilde{\mathcal{B}}_{z} W, \quad W_{0}(\mathbf{x}, \mathbf{p}) \in L^{2}\left(\mathbb{R}^{2 d}\right),
$$

where $\widetilde{\mathcal{B}}_{z}$ is the operator-valued Brownian motion with the spatial covariance operator $\mathcal{Q}^{(\eta, \rho)}(\mathbf{x}, \mathbf{y})$ defined by

$$
\begin{aligned}
\mathcal{Q}^{(\eta, \rho)}(\theta \otimes \theta)(\mathbf{x}, \mathbf{p}, \mathbf{y}, \mathbf{q})=\int & e^{i \mathbf{q}^{\prime} \cdot(\mathbf{x}-\mathbf{y})} \Phi_{(\eta, \rho)}\left(0, \mathbf{q}^{\prime}\right) \gamma^{-2} \\
& \times\left[\theta\left(\mathbf{x}, \mathbf{p}-\gamma \mathbf{q}^{\prime} / 2\right)-\theta\left(\mathbf{x}, \mathbf{p}+\gamma \mathbf{q}^{\prime} / 2\right)\right] \\
& \times\left[\theta\left(\mathbf{y}, \mathbf{q}-\gamma \mathbf{q}^{\prime} / 2\right)-\theta\left(\mathbf{y}, \mathbf{q}+\gamma \mathbf{q}^{\prime} / 2\right)\right] d \mathbf{q}^{\prime}, \\
\mathcal{Q}_{0}^{(\eta, \rho)} \theta(\mathbf{x}, \mathbf{p})=\int & \Phi_{(\eta, \rho)}(0, \mathbf{q}) \gamma^{-2}[\theta(\mathbf{x}, \mathbf{p}+\gamma \mathbf{q})+\theta(\mathbf{x}, \mathbf{p}-\gamma \mathbf{q})-2 \theta(\mathbf{x}, \mathbf{p})] d \mathbf{q}
\end{aligned}
$$

for $0 \leq \eta \leq \rho \leq \infty[13]$.

The $n$-point correlation functions of the Wigner distribution play an important role in applications. It is given by

$$
W^{(n)}\left(z, \mathbf{x}_{1}, \mathbf{p}_{1}, \ldots, \mathbf{x}_{n}, \mathbf{p}_{n}\right) \equiv \mathbb{E}\left[W\left(z, \mathbf{x}_{1}, \mathbf{p}_{1}\right) \cdots W\left(z, \mathbf{x}_{n}, \mathbf{p}_{n}\right)\right]
$$

and solves in this case the equation

$$
\begin{aligned}
& \frac{\partial W^{(n)}}{\partial z}+\frac{1}{\tilde{k}} \sum_{j=1}^{n} \mathbf{p}_{j} \cdot \nabla_{\mathbf{x}_{j}} W^{(n)} \\
& =\frac{\tilde{k}^{2}}{4} \sum_{j}^{n} \mathcal{Q}_{0}\left(\mathbf{x}_{j}, \mathbf{p}_{j}\right) W^{(n)}+\frac{\tilde{k}^{2}}{4} \sum_{\substack{j, k=1 \\
j \neq k}}^{n} \mathcal{Q}\left(\mathbf{x}_{j}, \mathbf{p}_{j}, \mathbf{x}_{k}, \mathbf{p}_{k}\right) W^{(n)},
\end{aligned}
$$

where $\mathcal{Q}\left(\mathbf{x}_{j}, \mathbf{p}_{j}, \mathbf{x}_{k}, \mathbf{p}_{k}\right)$ is the same operator $\mathcal{Q}$ defined in (3.24) acting on the two sets of phase-space variables $\left(\mathbf{x}_{j}, \mathbf{p}_{j}\right)$ and $\left(\mathbf{x}_{k}, \mathbf{p}_{k}\right)$ :

$$
\begin{aligned}
\mathcal{Q}\left(\mathbf{x}_{j}, \mathbf{p}_{j}, \mathbf{x}_{k}, \mathbf{p}_{k}\right) W^{(n)}\left(z, \mathbf{x}_{1}, \mathbf{p}_{1}, \ldots, \mathbf{x}_{n}, \mathbf{p}_{n}\right) \\
=\mathbb{E}\left\{\prod_{i \neq j, k} W\left(\mathbf{x}_{i}, \mathbf{p}_{i}\right) \int e^{i \mathbf{q}^{\prime} \cdot\left(\mathbf{x}_{j}-\mathbf{x}_{k}\right)} \Phi_{(\eta, \infty)}\left(0, \mathbf{q}^{\prime}\right) \gamma^{-2}\right. \\
\times\left[W\left(\mathbf{x}_{j}, \mathbf{p}_{j}-\gamma \mathbf{q} / 2\right)-W\left(\mathbf{x}_{j}, \mathbf{p}_{j}+\gamma \mathbf{q} / 2\right)\right] \\
\left.\times\left[W\left(\mathbf{x}_{k}, \mathbf{p}_{k}-\gamma \mathbf{q} / 2\right)-W\left(\mathbf{x}_{k}, \mathbf{p}_{k}+\gamma \mathbf{q} / 2\right)\right] d \mathbf{q}\right\} .
\end{aligned}
$$

An advantage of the Wigner-Itô formulation is that the moment equation (3.25) of order $n$ seems to be more tractable than (3.17) of order $2 n$. This will be seen clearly in the case of $n=1$ below. 
Another advantage of working with the Wigner-Itô equation is that we can use it to evolve any $L^{2}\left(\mathbb{R}^{2 d}\right)$ initial data, be it the cross Wigner distribution

$$
W\left(\Psi_{1}, \Psi_{2}\right)(\mathbf{x}, \mathbf{p})=\frac{1}{(2 \pi)^{d}} \int e^{-i \mathbf{p} \cdot \mathbf{y}} \Psi_{1}(\mathbf{x}+\gamma \mathbf{y} / 2) \overline{\Psi_{2}}(\mathbf{x}-\gamma \mathbf{y} / 2) d \mathbf{y}
$$

or the Wigner distribution of the mixed state

$$
W_{\text {mix }}(\mathbf{x}, \mathbf{p})=\int W\left(\Psi_{\alpha}, \Psi_{\alpha}\right)(\mathbf{x}, \mathbf{p}) d \mu(\alpha),
$$

where $\mu(\alpha)$ is a probability density function describing the mixture.

Note that the Wigner distribution may be missing an overall phase factor. Both $\Psi^{\varepsilon}$ and $\exp (i \phi(z)) \Psi^{\varepsilon}$ for $\phi$ a $z$-dependent phase give rise to the same Wigner distribution. Thus, the Wigner distribution erases the overall phase factor that is a function of $z$ alone and may affect the travel time statistics. In applications to time reversal, however, such a phase factor is not present.

In the case of geometrical optics approximation $\gamma \rightarrow 0$, the Wigner equation becomes the Liouville equation with a random potential $\nu(z, \mathbf{x})[27,13]$. We have the analogous convergence of the weak solution to the Gaussian white-noise model for the Liouville equation [13]. The Gaussian white-noise model for the Liouville equation with a smooth potential has been analyzed in [36].

The results in the rest of the paper will be mainly concerned with the solutions of the moment equation (3.25), in particular $n=1$, for $\rho=\infty$, i.e., a negligible inner scale.

4. Forward propagation in random media. In this section we analyze the transmitted wave field. First, in section 4.1 we compute the mean of the transmitted field when it is modeled in terms of the parabolic wave equation. The wave propagating through the turbulent medium will in general be strongly fluctuating due to the refractive turbulence. In section 4.2 we characterize the second moment of the wave field, the mutual coherence function that we can use to analyze these fluctuations. In section 4.3 we use the mutual coherence function to analyze the spreading of the wave, and in section 4.4 we examine the coherence length. The coherence length corresponds to a correlation length for the the wave field and relates to the spatial scale of the scintillations.

4.1. Transmitted pulse. We write the transmitted signal at $z$ in the form

$$
u(\tau, \mathbf{x}, z)=\int \hat{g}_{0}(\tilde{k}) \Psi(z, \mathbf{x}, \tilde{k}) e^{i \tilde{k}(\nu z-\tau)} d \tilde{k}+\text { c.c. }
$$

where

$$
\tau=c_{0} k_{0} t, \quad \nu=k_{0} L_{z},
$$

and $\Psi$ solves (3.15) in the white-noise limit with initial data

$$
\Psi(0, \mathbf{x}, \tilde{k})=\Psi_{0}(\mathbf{x}) .
$$

Here $\tau$ is the mean propagation distance and $\nu$ the reference length in the propagation direction, both in the unit of the wave length. Note that we use the convention

$$
\begin{aligned}
\hat{\psi}(\mathbf{w}) & =\frac{1}{(2 \pi)^{d}} \int e^{-i \mathbf{w} \cdot \mathbf{x}} \psi(\mathbf{x}) d \mathbf{x}, \\
\psi(\mathbf{x}) & =\int e^{i \mathbf{w} \cdot \mathbf{x}} \hat{\psi}(\mathbf{w}) d \mathbf{w}
\end{aligned}
$$


for the Fourier transform in space and

$$
\begin{aligned}
\hat{f}(\omega) & =\frac{1}{2 \pi} \int e^{i \omega t} f(t) d t \\
f(t) & =\int e^{-i \omega t} \hat{f}(\omega) d \omega
\end{aligned}
$$

for the Fourier transform in time.

Consider first the case with a homogeneous medium with $V \equiv 0$. The wave field is then deterministic and solves

$$
2 i \tilde{k} \frac{\partial \Psi^{0}}{\partial z}+\gamma \Delta \Psi^{0}=0
$$

Thus, we find $(d=2)$

$$
\Psi^{0}(z, \mathbf{x} ; \tilde{k})=-\frac{i \tilde{k}}{2 \pi z \gamma} \int \Psi_{0}(\mathbf{y}) \exp \left[i \tilde{k}(\mathbf{x}-\mathbf{y})^{2} /(2 \gamma z)\right] d \mathbf{y} .
$$

Observe that if $\Psi_{0}$ is narrowly supported, then the magnitude of $\Psi^{0}$ decays slowly in the lateral coordinates $\mathbf{x}$. The transmitted wave pulse is given by (4.1) with $\Psi$ replaced by $\Psi^{0}$ :

$$
u_{0}(\tau, \mathbf{x}, z)=\int \hat{g}_{0}(\tilde{k}) \Psi^{0}(z, \mathbf{x} ; \tilde{k}) e^{i \tilde{k}(\tau-\nu z)} d \tilde{k}+\text { c.c. }
$$

Consider next the random case. The coherent wave field $M_{1}=\mathbb{E}[\Psi]$ solves

$$
\frac{\partial M_{1}}{\partial z}=\frac{i \gamma}{2 \tilde{k}} \Delta_{\mathbf{x}} M_{1}-\frac{\tilde{k}^{2}}{4 \gamma^{2}} \Gamma_{0}^{(\eta, \infty)} M_{1}
$$

whose solution is given by

$$
M_{1}(z, \mathbf{x} ; \tilde{k})=-\frac{i \tilde{k}}{2 \pi z \gamma} \exp \left[-\Gamma_{0}^{(\eta, \infty)} \tilde{k}^{2} z /\left(4 \gamma^{2}\right)\right] \int \Psi_{0}(\mathbf{y}) \exp \left[i \tilde{k}(\mathbf{x}-\mathbf{y})^{2} /(2 \gamma z)\right] d \mathbf{y}
$$

It follows then the expression for the mean transmitted pulse,

$$
\mathbb{E}[u](\tau, \mathbf{x}, z)=\left[u_{0}(\cdot, \mathbf{x}, z) * \mathcal{N}_{\sigma}(\cdot)\right](\tau),
$$

where

$$
\begin{aligned}
\mathcal{N}_{\sigma}(\tau) & \equiv \frac{e^{-\tau^{2} /\left(2 \sigma^{2}\right)}}{\sqrt{2 \pi \sigma^{2}}}, \\
\sigma^{2} & =\frac{z \Gamma_{0}^{(\eta, \infty)}}{2 \gamma^{2}} .
\end{aligned}
$$

The formula (4.6) is, of course, due to the fact that $\sigma$ is independent of the offset $\mathbf{x}$. It follows that the multiscale random fluctuations in the medium has the effect of smearing the signal in time through convolution with a Gaussian pulse of square width $\sigma$. It results in the loss of coherence of the wave field even though the total wave energy is conserved. This loss of coherence is inversely related to the Fresnel number $\gamma$, and when $\gamma$ is small the transmitted pulse is typically supported on the time scale $\sqrt{z} / \gamma$. 
4.2. Mutual coherence functions. Define the two-point correlation function or the space-time mutual coherence function of the transmitted field in (4.1) as

$$
\begin{aligned}
& \Gamma\left(z, \mathbf{x}_{1}, \mathbf{x}_{2} ; \tau_{1}, \tau_{2}\right)=\mathbb{E}\left[u\left(\tau_{1}, z, \mathbf{x}_{1}\right) \overline{u\left(\tau_{2}, z, \mathbf{x}_{2}\right)}\right] \\
& =\int \hat{g}_{0}\left(\tilde{k}_{1}\right) \overline{\hat{g}_{0}\left(\tilde{k}_{2}\right)} \mathbb{E}\left[\Psi\left(z, \mathbf{x}_{1}, \tilde{k}_{1}\right) \overline{\Psi\left(z, \mathbf{x}_{2}, \tilde{k}_{2}\right)}\right] e^{i\left(\tilde{k}_{1} \tau_{1}-\tilde{k}_{2} \tau_{2}\right)} d \tilde{k}_{1} d \tilde{k}_{2} .
\end{aligned}
$$

The quantity

$$
\mathbb{E}\left[\Psi\left(z, \mathbf{x}_{1}, \tilde{k}_{1}\right) \overline{\Psi\left(z, \mathbf{x}_{2}, \tilde{k}_{2}\right)}\right]
$$

is the two frequency mutual coherence function where a random phase factor corresponding to a travel time correction will play an important role (see (A.10) in Appendix A.1). This issue is beyond the scope of the present paper.

Instead we assume that $g_{0}$ is narrowly supported around the wave number $\tilde{k}$ so that

$$
\Gamma\left(z, \mathbf{x}_{1}, \mathbf{x}_{2} ; \tau_{1}, \tau_{2}\right) \sim c \mathbb{E}\left[\Psi\left(z, \mathbf{x}_{1}, \tilde{k}\right) \overline{\Psi\left(z, \mathbf{x}_{2}, \tilde{k}\right)}\right] e^{i \tilde{k}\left(\tau_{1}-\tau_{2}\right)},
$$

where $c$ is a scaling constant. Therefore, the mutual coherence function

$$
M_{2}\left(z, \mathbf{x}_{1}, \mathbf{x}_{2} ; \tilde{k}\right)=\mathbb{E}\left[\Psi\left(z, \mathbf{x}_{1}, \tilde{k}\right) \overline{\Psi\left(z, \mathbf{x}_{2}, \tilde{k}\right)}\right]
$$

is needed to describe wave field fluctuations. Note the difference between $M_{2}$ and $F^{(2)}$ as given in (3.17) due to the complex conjugation.

In this section we consider the same-point mutual coherence function in the whitenoise limit

$$
\begin{aligned}
M_{2}(z, \mathbf{x}, \mathbf{x} ; \tilde{k}) & =\int \mathbb{E}[W(z, \mathbf{x}, \mathbf{p} ; \tilde{k})] d \mathbf{p} \\
& =\int W^{(1)}(z, \mathbf{x}, \mathbf{p} ; \tilde{k}) d \mathbf{p},
\end{aligned}
$$

which is the ensemble-averaged wave energy density in $\mathbf{x}$.

Similarly, we consider the mean wave energy density in $\mathbf{p}$,

$$
\begin{aligned}
\hat{M}_{2}(z, \mathbf{p}, \mathbf{p} ; \tilde{k}) & \equiv \mathbb{E}\left[|\hat{\Psi}(z, \mathbf{p}, \tilde{k})|^{2}\right] \\
& =\left(\frac{\gamma}{2 \pi}\right)^{d} \int \mathbb{E}[W(z, \mathbf{x}, \gamma \mathbf{p} ; \tilde{k})] d \mathbf{x} \\
& =\left(\frac{\gamma}{2 \pi}\right)^{d} \int W^{(1)}(z, \mathbf{x}, \gamma \mathbf{p} ; \tilde{k}) d \mathbf{x},
\end{aligned}
$$

where $\hat{\Psi}$ is the Fourier transform of $\Psi$ in the lateral spatial variables. $\hat{M}_{2}$ is the ensemble-averaged wave energy density in the transverse wavevector $\mathbf{p}$. The following scaled version $\tilde{M}_{2}$ of $\hat{M}_{2}$ will also be useful in our analysis:

$$
\begin{aligned}
\tilde{M}_{2}(z, \mathbf{p}, \mathbf{p} ; \tilde{k}) & =\hat{M}_{2}\left(z, \frac{\tilde{k} \mathbf{p}}{\gamma z}, \frac{\tilde{k} \mathbf{p}}{\gamma z} ; \tilde{k}\right) \\
& =\left(\frac{\gamma}{2 \pi}\right)^{d} \int \mathbb{E}\left[W\left(z, \mathbf{x}, \frac{\tilde{k} \mathbf{p}}{z} ; \tilde{k}\right)\right] d \mathbf{x},
\end{aligned}
$$


which can be interpreted as the ensemble-averaged image field on the spectral plane of a lens with $\mathbf{p}$ as the spatial frequency [7].

Using (3.25) we find that $W^{(1)}=W^{(1)}(z, \mathbf{x}, \mathbf{p})$ solves

$$
\frac{\partial W^{(1)}}{\partial z}+\frac{1}{\tilde{k}} \mathbf{p} \cdot \nabla_{\mathbf{x}} W^{(1)}=\overline{\mathcal{Q}}_{0} W^{(1)}
$$

with

$$
\overline{\mathcal{Q}}_{0} W^{(1)}=\frac{\tilde{k}^{2}}{4 \gamma^{2}} \int \Phi_{(\eta, \infty)}(0, \mathbf{q})\left(-2 W^{(1)}(\mathbf{p})+W^{(1)}(\mathbf{p}+\gamma \mathbf{q})+W^{(1)}(\mathbf{p}-\gamma \mathbf{q})\right) d \mathbf{q}
$$

for $\eta=0, H \in(0,1 / 2)$ or $\eta>0, H \in(0,1)$, which, unlike the second moment equation (3.17) for $\Psi$, is readily solvable. The Green's function $G_{W}^{(1)}$ solving (4.12) is given by

$$
\begin{aligned}
G_{W}^{(1)}(z, \mathbf{x}, \mathbf{p} ; \overline{\mathbf{x}}, \overline{\mathbf{p}})=\frac{1}{(2 \pi)^{2 d}} \int & e^{i(\mathbf{q} \cdot(\mathbf{x}-\overline{\mathbf{x}})-\mathbf{y} \cdot(\mathbf{p}-\overline{\mathbf{p}})-z \mathbf{q} \cdot \overline{\mathbf{p}} / \tilde{k})} \\
& \times \exp \left[-\tilde{k}^{2} /\left(2 \gamma^{2}\right) \int_{0}^{z} D_{*}(\gamma(\mathbf{y}+\mathbf{q}(s-z) / \tilde{k})) d s\right] d \mathbf{y} d \mathbf{q},
\end{aligned}
$$

where the structure function $D_{*}$ of the white-noise medium is defined by

$$
D_{*}(\mathbf{x})=\int \Phi_{(\eta, \infty)}(0, \mathbf{q})\left[1-e^{i \mathbf{x} \cdot \mathbf{q}}\right] d \mathbf{q}
$$

for $\eta=0, H \in(0,1 / 2)$ or $\eta>0, H \in(0,1)$. The structure function $D_{*}$ is not well defined for $\eta=0, H=[1 / 2,1)$. It is different from the original structure function of the refractive index field $D_{n}$ given in (2.2) in that only the zeroth mode in the longitudinal direction contributes to the integral over the transverse directions. For simplicity of notation we continue to assume an isotropic medium.

The main property of $D_{*}$ we need in what follows is the short distance asymptotic

$$
D_{*}(r) \approx C_{*}^{2} r^{2 H_{*}} \quad \text { for } r \ll 1 / \eta,
$$

where the effective Hölder exponent $H_{*}$ is given by

$$
H_{*}= \begin{cases}H+1 / 2 & \text { for } H \in(0,1 / 2), \\ 1 & \text { for } H \in(1 / 2,1],\end{cases}
$$

and the structure parameter $C_{*}^{2}$ is proportional to $4 \times 0.033 C_{n}^{2}$. The effective Hölder exponent $H_{*}$ is always bigger than $1 / 2$, and hence the white-noise medium has a "persistent" transverse correlation. and $\tilde{M}_{2}$ :

Using this Green's function we find the following explicit expression for $M_{2}, \hat{M}_{2}$,

$$
\begin{aligned}
& M_{2}(z, \mathbf{x}, \mathbf{x} ; \tilde{k}) \\
& =\frac{1}{(2 \pi)^{3 d}} \int e^{-i \overline{\mathbf{p}} \cdot \mathbf{y}} \Psi_{0}\left(\overline{\mathbf{x}}+\frac{\gamma \mathbf{y}}{2}\right) \overline{\Psi_{0}\left(\overline{\mathbf{x}}-\frac{\gamma \mathbf{y}}{2}\right)} e^{i(\mathbf{w} \cdot(\mathbf{x}-\overline{\mathbf{x}})+\mathbf{r} \cdot(\mathbf{p}-\overline{\mathbf{p}})-z \mathbf{w} \cdot \overline{\mathbf{p}} / \tilde{k})} \\
& \quad \times \exp \left[-\tilde{k}^{2} /\left(2 \gamma^{2}\right) \int_{0}^{z} D_{*}(\gamma(\mathbf{r}+\mathbf{w}(z-s)) / \tilde{k}) d s\right] d \mathbf{y} d \mathbf{r} d \mathbf{w} d \overline{\mathbf{x}} d \overline{\mathbf{p}} d \mathbf{p}
\end{aligned}
$$




$$
\begin{aligned}
=\frac{1}{(2 \pi)^{d}} \int & \Psi_{0}\left(\overline{\mathbf{x}}-\frac{\gamma z \mathbf{w}}{2 \tilde{k}}\right) \overline{\Psi_{0}\left(\overline{\mathbf{x}}+\frac{\gamma z \mathbf{w}}{2 \tilde{k}}\right)} e^{i \mathbf{w} \cdot(\mathbf{x}-\overline{\mathbf{x}})} \\
& \times \exp \left[-\tilde{k}^{2} /\left(2 \gamma^{2}\right) \int_{0}^{z} D_{*}(\gamma \mathbf{w}(z-s) / \tilde{k}) d s\right] d \mathbf{w} d \overline{\mathbf{x}},
\end{aligned}
$$

$$
\begin{aligned}
& \hat{M}_{2}(z, \mathbf{p}, \mathbf{p} ; \tilde{k}) \\
& =\frac{\gamma^{d}}{(2 \pi)^{4 d}} \int e^{-i \overline{\mathbf{p}} \cdot \mathbf{y}} \Psi_{0}(\overline{\mathbf{x}}+\gamma \mathbf{y} / 2) \overline{\Psi_{0}(\overline{\mathbf{x}}-\gamma \mathbf{y} / 2)} e^{i \mathbf{w} \cdot(\mathbf{x}-\overline{\mathbf{x}})} e^{i \mathbf{r} \cdot(\gamma \mathbf{p}-\overline{\mathbf{p}})} e^{-z \mathbf{w} \cdot \mathbf{p} / \tilde{k}} \\
& \quad \times \exp \left[-\tilde{k}^{2} /\left(2 \gamma^{2}\right) \int_{0}^{z} D_{*}(\gamma(\mathbf{r}+\mathbf{w} \cdot(z-s)) / \tilde{k}) d s\right] d \mathbf{y} d \mathbf{r} d \mathbf{w} d \overline{\mathbf{x}} d \overline{\mathbf{p}} d \mathbf{x} \\
& =\left(\frac{\gamma}{(2 \pi)^{2}}\right)^{d} \int \Psi_{0}(\overline{\mathbf{x}}+\gamma \mathbf{y} / 2) \overline{\Psi_{0}(\overline{\mathbf{x}}-\gamma \mathbf{y} / 2)} e^{-i \gamma \mathbf{p} \cdot \mathbf{y}} \exp \left[-\tilde{k}^{2} /\left(2 \gamma^{2}\right) z D_{*}(-\gamma \mathbf{y})\right] d \mathbf{y} d \overline{\mathbf{x}} \\
& =\left(\frac{1}{2 \pi}\right)^{2 d} \int \Psi_{0}(\overline{\mathbf{x}}+\mathbf{y} / 2) \overline{\Psi_{0}(\overline{\mathbf{x}}-\mathbf{y} / 2)} e^{-i \mathbf{p} \cdot \mathbf{y}} \exp \left[-\tilde{k}^{2} /\left(2 \gamma^{2}\right) z D_{*}(-\mathbf{y})\right] d \mathbf{y} d \overline{\mathbf{x}}, \\
& (4.20) \\
& \tilde{M}_{2}(z, \mathbf{p}, \mathbf{p} ; \tilde{k}) \\
& =\left(\frac{1}{2 \pi}\right)^{2 d} \int \Psi_{0}(\overline{\mathbf{x}}+\mathbf{y} / 2) \overline{\Psi_{0}(\overline{\mathbf{x}}-\mathbf{y} / 2)} e^{-i \tilde{k} \mathbf{p} \cdot \mathbf{y} /(\gamma z)} \exp \left[-\tilde{k}^{2} /\left(2 \gamma^{2}\right) z D_{*}(-\mathbf{y})\right] d \mathbf{y} d \overline{\mathbf{x}} .
\end{aligned}
$$

To see the effect of the turbulent medium on the transmitted field more clearly it is convenient to use the Gaussian source or pupil function

$$
\Psi_{0}(\mathbf{x})=\exp \left[-\frac{|\mathbf{x}|^{2}}{2 \alpha^{2}}\right],
$$

with $\alpha>0$ corresponding to the aperture of the incident beam. With (4.21) the mutual coherence functions have the following forms:

$$
\begin{aligned}
& M_{2}(z, \mathbf{x}, \mathbf{x} ; \tilde{k}) \\
& =\frac{1}{(2 \pi)^{d}} \int e^{-|\overline{\mathbf{x}}+\gamma z \mathbf{w} /(2 \tilde{k})|^{2} /\left(2 \alpha^{2}\right)} e^{-|\overline{\mathbf{x}}-\gamma z \mathbf{w} /(2 \tilde{k})|^{2} /\left(2 \alpha^{2}\right)} \\
& \quad \times e^{i \mathbf{w} \cdot(\mathbf{x}-\overline{\mathbf{x}})} \exp \left[-\tilde{k}^{2} /\left(2 \gamma^{2}\right) \int_{0}^{z} D_{*}(\gamma \mathbf{w}(z-s) / \tilde{k}) d s\right] d \mathbf{w} d \overline{\mathbf{x}} \\
& =\left(\frac{\alpha}{2 \sqrt{\pi}}\right)^{d} \int e^{-|\mathbf{w}|^{2}\left[\alpha^{2} / 4+\gamma^{2} z^{2} /\left(4 \tilde{k}^{2} \alpha^{2}\right)\right]} \exp \left[-\tilde{k}^{2} /\left(2 \gamma^{2}\right) \int_{0}^{z} D_{*}(\gamma \mathbf{w} s / \tilde{k}) d s\right] e^{i \mathbf{w} \cdot \mathbf{x}} d \mathbf{w},
\end{aligned}
$$

$$
\hat{M}_{2}(z, \mathbf{p}, \mathbf{p} ; \tilde{k})=\left(\frac{\alpha}{2 \sqrt{\pi}}\right)^{d} \int e^{-|\mathbf{y}|^{2} /\left(4 \alpha^{2}\right)} \exp \left[-\tilde{k}^{2} /\left(2 \gamma^{2}\right) z D_{*}(-\mathbf{y})\right] e^{-i \mathbf{p} \cdot \mathbf{y}} d \mathbf{y},
$$

$$
\begin{aligned}
\tilde{M}_{2}(z, \mathbf{p}, \mathbf{p} ; \tilde{k})=\left(\frac{\alpha \gamma z}{2 \tilde{k} \sqrt{\pi}}\right)^{d} \int & e^{-|\mathbf{y}|^{2} \gamma^{2} z^{2} /\left(4 \tilde{k}^{2} \alpha^{2}\right)} \\
& \times \exp \left[-\tilde{k}^{2} /\left(2 \gamma^{2}\right) z D_{*}(-\gamma z \mathbf{y} / \tilde{k})\right] e^{-i \mathbf{p} \cdot \mathbf{y}} d \mathbf{y}
\end{aligned}
$$


In view of (4.22), (4.23), and (4.24) we see that the transmission of radiative energy in either position or wavevector space is a convolution process, and we can associate the transfer functions

$$
\begin{aligned}
& T(z, \mathbf{y})=\exp \left[-\tilde{k}^{2} /\left(2 \gamma^{2}\right) \int_{0}^{z} D_{*}(-\gamma \mathbf{y} s / \tilde{k}) d s\right], \\
& \hat{T}(z, \mathbf{y})=\exp \left[-\tilde{k}^{2} /\left(2 \gamma^{2}\right) z D_{*}(-\mathbf{y})\right], \\
& \tilde{T}(z, \mathbf{y})=\exp \left[-\tilde{k}^{2} /\left(2 \gamma^{2}\right) z D_{*}(-\gamma z \mathbf{y} / \tilde{k})\right]
\end{aligned}
$$

to the turbulent medium depending on which of the three coherence functions is used. Note that the parameterization in $\tilde{M}_{2}$ has been chosen so that its scaling is analogous to that of $M_{2}$. By analyzing these transfer functions one can determine when the effects of the turbulence become important.

4.3. High-frequency beam-spreading asymptotics. The support of the mutual coherence function determines the lateral support of the wave energy. We consider a high-frequency asymptotics scaling with $z \gamma / \tilde{k} \ll \eta^{-1}$ for various media models. For low frequency waves $z \gamma \gg \tilde{k} / \eta$ the pulse is not much affected by the random medium, and the spreading is roughly the same as in the deterministic case.

4.3.1. Homogeneous medium. In this case with $D_{*} \equiv 0$ the transmitted energy envelope has the Gaussian form

$$
M_{2}(z, \mathbf{x}, \mathbf{x}, \tilde{k})=\left(\frac{\alpha}{\sqrt{2} s_{0}}\right)^{d} \exp \left(-\frac{|\mathbf{x}|^{2}}{2 s_{0}^{2}}\right)
$$

and is supported on the scale of the position spread

$$
s_{0}(z, \tilde{k})=\frac{1}{2} \sqrt{\alpha^{2}+\left(\frac{\gamma z}{\tilde{k} \alpha}\right)^{2}} .
$$

In contrast,

$$
\begin{aligned}
& \hat{M}_{2}(z, \mathbf{p}, \mathbf{p} ; \tilde{k})=\left(\frac{\alpha}{\sqrt{2} \hat{s}_{0}}\right)^{d} \exp \left[-\frac{|\mathbf{p}|^{2}}{2 \hat{s}_{0}^{2}}\right], \\
& \tilde{M}_{2}(z, \mathbf{p}, \mathbf{p} ; \tilde{k})=\left(\frac{\gamma z \alpha}{\sqrt{2} \tilde{k} \tilde{s}_{0}}\right)^{d} \exp \left[-\frac{|\mathbf{p}|^{2}}{2 \tilde{s}_{0}^{2}}\right]
\end{aligned}
$$

are supported on the transverse wavevector spreading scales

$$
\begin{aligned}
& \hat{s}_{0}=\frac{1}{\sqrt{2} \alpha}, \\
& \tilde{s}_{0}=\frac{\gamma z}{\sqrt{2} \tilde{k} \alpha} .
\end{aligned}
$$

One sees from (4.26) that the optimal source size $\alpha=\sqrt{\gamma z / \tilde{k}}$ minimizes the spread in position giving the smallest spreading factor,

$$
\sqrt{2 \gamma z / \tilde{k}}=\sqrt{2} \frac{\gamma z}{\alpha \tilde{k}}
$$

and from (4.29) that there is no limitation on how focused the wave energy can be in the wavevector or spatial frequency. 
4.3.2. Smooth white-noise medium. The medium is assumed to have a finite, lateral correlation length $1 / \eta<\infty$ such that $D_{*} \in C^{2}(\mathbb{R})$ with $D_{*}{ }^{\prime}(0)=0$, $\left|D_{*}{ }^{\prime \prime}(0)\right|<\infty$. When $z \gamma \ll \tilde{k} / \eta$ the transmitted energy envelope has approximately the same form as above,

$$
M_{2}(z, \mathbf{x}, \mathbf{x} ; \tilde{k}) \approx\left(\frac{\alpha}{\sqrt{2} s_{*}}\right)^{d} \exp \left[-\frac{|\mathbf{x}|^{2}}{2 s_{*}^{2}}\right], \quad \alpha \gg \gamma,
$$

with, however, a different spread:

$$
s_{*}=\sqrt{s_{0}^{2}+\frac{D_{*}^{\prime \prime}(0) z^{3}}{6}} .
$$

In contrast,

$$
\begin{aligned}
& \hat{M}_{2}(z, \mathbf{p}, \mathbf{p} ; \tilde{k}) \approx\left(\frac{\alpha}{\sqrt{2} \hat{s}_{*}}\right)^{d} \exp \left[-\frac{|\mathbf{p}|^{2}}{2 \hat{s}_{*}^{2}}\right], \\
& \tilde{M}_{2}(z, \mathbf{p}, \mathbf{p} ; \tilde{k}) \approx\left(\frac{\gamma z \alpha}{\sqrt{2} \tilde{k}_{\tilde{s}_{*}}}\right)^{d} \exp \left[-\frac{|\mathbf{p}|^{2}}{2 \tilde{s}_{*}^{2}}\right]
\end{aligned}
$$

with

$$
\begin{aligned}
& \hat{s}_{*}=\sqrt{\hat{s}_{0}^{2}+\frac{\tilde{k}^{2} z D_{*}{ }^{\prime \prime}(0)}{2 \gamma^{2}},} \\
& \tilde{s}_{*}=\gamma z \hat{s}_{*} / \tilde{k} .
\end{aligned}
$$

Note that in all cases the influence of the smooth medium fluctuations on the spreading depends on the value of $D_{*}{ }^{\prime \prime}$ which reflects the roughness of the random medium.

4.3.3. Rough white-noise medium. The medium is assumed to have a finite, lateral correlation length $1 / \eta$ such that $z \gamma \ll \tilde{k} / \eta$, and we also assume that $D_{*} \in C^{1}(0, \infty)$ with $D_{*}{ }^{\prime}\left(0^{+}\right) \neq 0$. Physically speaking, a rough medium typically has independent spatial increments. Then the transmitted energy envelope has approximately the form of the bivariate Cauchy distribution in the position as well as the wavevector variables:

$$
M_{2}(z, \mathbf{x}, \mathbf{x} ; \tilde{k}) \approx\left(\frac{\alpha}{2 s_{*}}\right)^{d}\left(\frac{1}{1+|\mathbf{x}|^{2} / s_{*}^{2}}\right)^{(d+1) / 2}, \quad d=2,
$$

with the medium-induced spread

$$
s_{*} \approx \frac{D_{*}^{\prime}\left(0^{+}\right) z^{2} \tilde{k}}{4 \gamma}
$$

moreover,

$$
\begin{aligned}
& \hat{M}_{2}(z, \mathbf{p}, \mathbf{p} ; \tilde{k}) \approx\left(\frac{\alpha}{\hat{s}_{*}}\right)^{d}\left(\frac{1}{1+|\mathbf{p}|^{2} / \hat{s}_{*}^{2}}\right)^{(d+1) / 2}, \\
& \tilde{M}_{2}(z, \mathbf{p}, \mathbf{p} ; \tilde{k}) \approx\left(\frac{\gamma z \alpha}{\tilde{k} \tilde{s}_{*}}\right)^{d}\left(\frac{1}{1+|\mathbf{p}|^{2} / \tilde{s}_{*}^{2}}\right)^{(d+1) / 2}
\end{aligned}
$$


with the medium-induced spreads

$$
\begin{aligned}
& \hat{s}_{*} \approx \frac{D_{*}{ }^{\prime}\left(0^{+}\right) z \tilde{k}^{2}}{2 \gamma^{2}}, \\
& \tilde{s}_{*} \approx \frac{D_{*}^{\prime}\left(0^{+}\right) z^{2} \tilde{k}}{2 \gamma} .
\end{aligned}
$$

The expressions (4.36), (4.38), and (4.39) yield heavy-tailed density functions whose variances are not well defined. This is a first manifestation of the effect of nonsmooth random fluctuations of the medium. Moreover, their first absolute moments

$$
\int|\mathbf{x}| M_{2}(z, \mathbf{x}, \mathbf{x}, \tilde{k}) d \mathbf{x}, \quad \int|\mathbf{p}| \hat{M}_{2}(z, \mathbf{p}, \mathbf{p}, \tilde{k}) d \mathbf{p}, \quad \int|\mathbf{p}| \tilde{M}_{2}(z, \mathbf{p}, \mathbf{p}, \tilde{k}) d \mathbf{p}
$$

are divergent logarithmically at $|\mathbf{x}|=\infty$ and $|\mathbf{p}|=\infty$, respectively. The first absolute moments, however, become convergent for the turbulent medium which is a natural interpolation between the smooth $\left(H_{*}=1\right)$ and the rough $\left(H_{*}=1 / 2\right)$ cases (see below). Hence we will ascribe to $s_{*}$ and $\hat{s}_{*}$ roughly the roles as the scales of the pulse in the position and transverse wavevector, respectively, to interpret another manifestation of the nonsmooth medium fluctuations, namely the wide spread of the pulse, in both the position and transverse wavevector for small $z \gamma \ll \tilde{k} / \eta$.

4.3.4. Turbulent medium. The power-law spectrum of the refractive index field has the Hölder exponent $H \in(0,1)$ as $\rho \rightarrow \infty$ and results in a white-noise medium with the effective Hölder exponent $H_{*} \in(1 / 2,1]$. The limiting case $H_{*}=1$ corresponds to the smooth case and $H_{*}=1 / 2$ to the rough case which then can be seen as having independent medium variations in the lateral directions.

When the effect of the turbulent medium dominates (see remarks after (4.54) and (4.60) for more elaboration on this), the wave energy envelope has the form in the position variables

$$
\begin{aligned}
M_{2}(z, \mathbf{x}, \mathbf{x} ; \tilde{k}) \approx & \left(\frac{C_{2} z^{-1-1 /\left(2 H_{*}\right)} \gamma^{1 / H_{*}-1} \alpha}{\sqrt{2 \pi}}\right)^{d} \\
& \times \exp \left[i C_{2} \mathbf{w} \cdot \mathbf{x} z^{-1-1 /\left(2 H_{*}\right)} \gamma^{1 / H_{*}-1}\right] \int e^{-|\mathbf{w}|^{2 H_{*}} / 2} d \mathbf{w}
\end{aligned}
$$

with

$$
C_{2}=\left(\frac{2 H_{*}+1}{C_{*}^{2} \tilde{k}^{2-2 H_{*}}}\right)^{1 /\left(2 H_{*}\right)}
$$

and the form in the transverse wavevector

$$
\begin{aligned}
\hat{M}_{2}(z, \mathbf{p}, \mathbf{p} ; \tilde{k}) & \approx\left(\frac{\alpha}{2 \sqrt{\pi}}\right)^{d} \int e^{-i \mathbf{p} \cdot \mathbf{y}} \exp \left[-\tilde{k}^{2} z C_{*}^{2}|\mathbf{y}|^{2 H_{*}} /\left(2 \gamma^{2}\right)\right] d \mathbf{y} \\
& \approx\left(\frac{\hat{C}_{2} z^{-1 /\left(2 H_{*}\right)} \alpha}{2 \sqrt{\pi}}\right)^{d} \int \exp \left[-i \hat{C}_{2} \mathbf{p} \cdot \mathbf{y} z^{-1 /\left(2 H_{*}\right)} \gamma^{1 / H_{*}}\right] e^{-|\mathbf{y}|^{2 H_{*} / 2}} d \mathbf{y},
\end{aligned}
$$




$$
\begin{aligned}
\tilde{M}_{2}(z, \mathbf{p}, \mathbf{p} ; \tilde{k}) & \approx\left(\frac{\alpha \gamma z}{2 \sqrt{\pi} \tilde{k}}\right)^{d} \int e^{-i \mathbf{p} \cdot \mathbf{y}} \exp \left[-\tilde{k}^{2-2 H_{*}} \gamma^{2 H_{*}-2} z^{2 H_{*}+1} C_{*}^{2}|\mathbf{y}|^{2 H_{*}} / 2\right] d \mathbf{y} \\
& \approx\left(\frac{\hat{C}_{2} z^{-1 /\left(2 H_{*}\right)} \alpha}{2 \sqrt{\pi}}\right)^{d} \int \exp \left[-i \tilde{k} \hat{C}_{2} \mathbf{p} \cdot \mathbf{y} z^{-1-1 /\left(2 H_{*}\right)} \gamma^{1 / H_{*}-1}\right] e^{-|\mathbf{y}|^{2 H_{*} / 2}} d \mathbf{y}
\end{aligned}
$$

with

$$
\hat{C}_{2}=\left(\tilde{k} C_{*}\right)^{-1 / H_{*}} .
$$

The integrals in (4.42), (4.43), and (4.44) give rise to Levy-like density functions which do not admit simple, explicit expressions. As in (4.36), (4.38), and (4.39), $M_{2}$, $\hat{M}_{2}$, and $\tilde{M}_{2}$ here have heavy tails with ill-defined variances for $H_{*}<1$. They do have, however, convergent first absolute moments which can be used to define the turbulence-induced spreads as

$$
\begin{gathered}
s_{*} \equiv \sqrt{\frac{\int|\mathbf{x}-\overline{\mathbf{x}}|^{2} \mathcal{F}[T]^{2}(z, \mathbf{x}) d \mathbf{x}}{\int \mathcal{F}[T]^{2}(z, \mathbf{x}) d \mathbf{x}}}<\infty, \quad \overline{\mathbf{x}} \equiv \frac{\int \mathbf{x} \mathcal{F}[T]^{2}(z, \mathbf{x}) d \mathbf{x}}{\int \mathcal{F}[T]^{2}(z, \mathbf{x}) d \mathbf{x}}, \\
\hat{s}_{*} \equiv \sqrt{\frac{\int|\mathbf{p}-\overline{\mathbf{p}}|^{2} \mathcal{F}[\hat{T}]^{2}(z, \mathbf{p}, \mathbf{p} ; \tilde{k}) d \mathbf{p}}{\int \mathcal{F}[\hat{T}]^{2}(z, \mathbf{p}) d \mathbf{p}}}<\infty, \quad \overline{\mathbf{p}} \equiv \frac{\int \mathbf{p} \mathcal{F}[\hat{T}]^{2}(z, \mathbf{p}) d \mathbf{p}}{\int \mathcal{F}[\hat{T}]^{2}(z, \mathbf{p}) d \mathbf{p}}, \\
\tilde{s}_{*} \equiv \sqrt{\frac{\int|\mathbf{q}-\gamma z \overline{\mathbf{p}} / \tilde{k}|^{2} \mathcal{F}[\tilde{T}]^{2}(z, \mathbf{q}) d \mathbf{q}}{\int \mathcal{F}[\tilde{T}]^{2}(z, \mathbf{q}) d \mathbf{q}}}=\frac{\gamma z \hat{s}_{*}}{\tilde{k}}
\end{gathered}
$$

where $\mathcal{F}[T]$ denotes the Fourier transform of $T$. The spreads $s_{*}, \hat{s}_{*}$, and $\tilde{s}_{*}$ are measures of the pulse's dispersion in position, transverse wavevector, and spatial frequency, respectively. The turbulence-induced spreads have the following asymptotics:

$$
\begin{aligned}
s_{*} & \sim C_{*}^{1 / H_{*}} \tilde{k}^{-1+1 / H_{*}} z^{1+1 /\left(2 H_{*}\right)} \gamma^{1-1 / H_{*}}, \\
\hat{s}_{*} & \sim C_{*}^{1 / H_{*}} \tilde{k}^{1 / H_{*}} z^{1 /\left(2 H_{*}\right)} \gamma^{-1 / H_{*}}, \\
\tilde{s}_{*} & \sim C_{*}^{1 / H_{*}} \tilde{k}^{-1+1 / H_{*}} z^{1+1 /\left(2 H_{*}\right)} \gamma^{1-1 / H_{*}}
\end{aligned}
$$

for $z \gamma \ll \tilde{k} / \eta$. The turbulence effect dominates when

$$
s_{*} \gg s_{0}, \quad \hat{s}_{*} \gg \hat{s}_{0}, \quad \tilde{s}_{*} \gg \tilde{s}_{0} .
$$

In particular, for the Kolmogorov spectrum $H_{*}=5 / 6$ the wave pulse scales as $z^{8 / 5} \gamma^{-1 / 5}$ in position and spatial frequency and $z^{3 / 5} \gamma^{-6 / 5}$ in transverse wavevector. Consider the case of vertical propagation through the atmosphere with $H=1 / 3$ and $\lambda=0.5 \mu \mathrm{m}$ and the altitude dependence of $C_{n}^{2}$ based on the data in [29]. This gives $5 \mathrm{~cm}$ as an estimate for $\tilde{s}_{*}$ at the altitude $h=10-15 \mathrm{~km}[23,24,25]$. The apparent smallness of the value is due to the smallness of the structure parameter $C_{*} \sim C_{n}$.

The spreads are related to the following notions of contrast or band-width defined as the ratio of the peak intensity to the total transmitted wave energy:

$$
\kappa_{*}=\frac{\sup _{\mathbf{x}} M_{2}(z, \mathbf{x}, \mathbf{x} ; \tilde{k})}{\int M_{2}(z, \mathbf{x}, \mathbf{x} ; \tilde{k}) d \mathbf{x}}, \quad \hat{\kappa}_{*}=\frac{\sup _{\mathbf{p}} \hat{M}_{2}(z, \mathbf{p}, \mathbf{p} ; \tilde{k})}{\int \hat{M}_{2}(z, \mathbf{p}, \mathbf{p} ; \tilde{k}) d \mathbf{p}}, \quad \tilde{\kappa}_{*}=\frac{\sup _{\mathbf{q}} \tilde{M}_{2}(z, \mathbf{q}, \mathbf{q} ; \tilde{k})}{\int \tilde{M}_{2}(z, \mathbf{q}, \mathbf{q} ; \tilde{k}) d \mathbf{q}} .
$$


It is easily seen that when (4.51) holds,

$$
\kappa_{*} \sim s_{*}^{-d}, \quad \hat{\kappa}_{*} \sim \hat{s}_{*}^{-d}, \quad \tilde{\kappa}_{*} \sim \tilde{s}_{*}^{-d} .
$$

The turbulent medium gives rise to an increasingly wider distribution, and thus smaller contrast, in both position and transverse wavevector, of the transmitted wave energy as $H$ and $\gamma$ decrease and as $C_{*}$ and $z$ increase.

4.3.5. Diversity exponent. Summarizing the above we have found that the spread takes place on the lateral scale in position and spatial frequency like $z^{1+\zeta / 2} \gamma^{1-\zeta}$ and in wavevector like $z^{\zeta / 2} \gamma^{-\zeta}$ with

$$
\zeta=\frac{1}{H_{*}} \in[1,2)
$$

which measures the roughness of the medium with $\zeta=2$ for the persistently correlated $(H>1 / 2)$ isotropic medium, $\zeta=1$ for the merely continuous $(H=0)$ isotropic medium, and $\zeta \in(1,2)$ for the antipersistently correlated $(H \in(0,1 / 2))$ isotropic medium. We shall refer to $\zeta$ as the diversity exponent.

The limit $H \geq 1 / 2$, corresponding to $H_{*}=1$, gives rise to a smooth white-noise medium while the limit $H \rightarrow 0$, corresponding to $H_{*} \rightarrow 1 / 2$, mimics the case of a rough medium. The Kolmogorov spectrum $H=1 / 3$ possesses the diversity exponent $\zeta=6 / 5$. We remark that an anisotropic medium which has the power-law spectrum (2.5) with $d=1$ in the two transverse dimensions and a finite correlation length in the direction of propagation gives rise to a white-noise model with the effective Hölder exponent $H_{*}=H \in(0,1)$ (i.e., $\zeta \in(1, \infty)$ ).

Alternatively, we can interpret the result by associating the spreading by turbulence with the notion of turbulence-induced entrance aperture by the relation

$$
\alpha_{*} \equiv \frac{\gamma z}{\tilde{k} \tilde{s}_{*}} \sim C_{*}^{-1 / H_{*}} \tilde{k}^{-1 / H_{*}} z^{-1 /\left(2 H_{*}\right)} \gamma^{1 / H_{*}}=C_{*}^{-\zeta} \tilde{k}^{-\zeta} z^{-\zeta / 2} \gamma^{\zeta}
$$

whose meaning can be appreciated by considering (4.29).

¿From (4.47) it follows that

$$
\alpha_{*}=\frac{1}{\hat{s}_{*}} .
$$

The turbulence effect becomes important when $\alpha_{*}<\alpha$ or, equivalently, when

$$
\hat{s}_{*} \alpha>1
$$

(cf. (4.30)). In the case of vertical propagation through the atmosphere with $H=1 / 3$ and $C_{n}^{2}$ based on the data [29] at the wavelength $\lambda=0.5 \mu \mathrm{m}$ and the altitude of up to 10 or $15 \mathrm{~km}$, the turbulence-induced entrance aperture $\alpha_{*}$ is never more than $20 \mathrm{~cm}$.

Because the turbulent medium with $H_{*} \in(1 / 2,1]$ provides a natural interpolation between the smooth randomness $\left(H_{*}=1\right)$ and the rough randomness $\left(H_{*}=1 / 2\right)$, in what follows we will focus on the calculation with $H_{*}$ and contrast it with the homogeneous case. We will comment on the limiting cases only when necessary.

4.4. Coherence lengths. We generalize the above calculations to the correlation at two different lateral points in this section. 
We define the centered coherence function in position and transverse wavevector

$$
\begin{aligned}
R_{2}(z, \mathbf{x}, \mathbf{y} ; \tilde{k}) & =\mathbb{E}[\Psi(z, \mathbf{x}+\mathbf{y} / 2 ; \tilde{k}) \overline{\Psi(z, \mathbf{x}-\mathbf{y} / 2 ; \tilde{k})}] \\
& =M_{2}(\mathbf{x}+\mathbf{y} / 2, \mathbf{x}-\mathbf{y} / 2)
\end{aligned}
$$

then

$$
\begin{aligned}
& R_{2}(z, \mathbf{x}, \mathbf{y} ; \tilde{k})=\int e^{i \mathbf{p} \cdot \mathbf{y} / \gamma} W^{(1)}(z, \mathbf{x}, \mathbf{p} ; \tilde{k}) d \mathbf{p} \\
& =\frac{1}{(2 \pi)^{3 d}} \int e^{i(\mathbf{p} \cdot \mathbf{y} / \gamma-\overline{\mathbf{p}} \cdot \overline{\mathbf{y}})} \Psi_{0}\left(\overline{\mathbf{x}}+\frac{\gamma \overline{\mathbf{y}}}{2}\right) \overline{\Psi_{0}\left(\overline{\mathbf{x}}-\frac{\gamma \overline{\mathbf{y}}}{2}\right)} e^{i(\mathbf{w} \cdot(\mathbf{x}-\overline{\mathbf{x}})+\mathbf{r} \cdot(\mathbf{p}-\overline{\mathbf{p}})-z \mathbf{w} \cdot \overline{\mathbf{p}} / \tilde{k})} \\
& \quad \times \exp \left[-\tilde{k}^{2} /\left(2 \gamma^{2}\right) \int_{0}^{z} D_{*}(\gamma(\mathbf{r}+\mathbf{w}(z-s) / \tilde{k})) d s\right] d \overline{\mathbf{y}} d \mathbf{r} d \mathbf{w} d \overline{\mathbf{x}} d \overline{\mathbf{p}} d \mathbf{p} \\
& =\frac{1}{(2 \pi)^{d}} \int \Psi_{0}(\overline{\mathbf{x}}+\mathbf{y} / 2-\gamma z \mathbf{w} /(2 \tilde{k})) \overline{\Psi_{0}(\overline{\mathbf{x}}-\mathbf{y} / 2+\gamma z \mathbf{w} /(2 \tilde{k}))} e^{i \mathbf{w} \cdot(\mathbf{x}-\overline{\mathbf{x}})} \\
& \quad \times \exp \left[-\tilde{k}^{2} /\left(2 \gamma^{2}\right) \int_{0}^{z} D_{*}(-\mathbf{y}+\gamma \mathbf{w}(z-s) / \tilde{k}) d s\right] d \mathbf{w} d \overline{\mathbf{x}} \\
& =\frac{1}{(2 \pi)^{d}} \int \Psi_{0}\left(\overline{\mathbf{x}}+\frac{\gamma z \mathbf{q}}{2 \tilde{k}}\right) \overline{\Psi_{0}\left(\overline{\mathbf{x}}-\frac{\gamma z \mathbf{q}}{2 \tilde{k}}\right)} e^{i(-\mathbf{q}+\tilde{k} \mathbf{y} /(\gamma z)) \cdot(\mathbf{x}-\overline{\mathbf{x}})} \\
& \quad \times \exp \left[-\tilde{k}^{2} /\left(2 \gamma^{2}\right) \int_{0}^{z} D_{*}(-s \mathbf{y} / z-\gamma \mathbf{q}(z-s) / \tilde{k}) d s\right] d \mathbf{q} d \overline{\mathbf{x}} .
\end{aligned}
$$

For the Gaussian initial data (4.21) we have

$$
\begin{aligned}
& \quad R_{2}(z, \mathbf{x}, \mathbf{y} ; \tilde{k}) \\
& =\left(\frac{\alpha}{\sqrt{2 \pi}}\right)^{d} \int e^{-|\mathbf{q}-\tilde{k} \mathbf{y} /(\gamma z)|^{2} /\left(4 \alpha^{2}\right)} e^{-\gamma^{2} z^{2}|\mathbf{q}|^{2} /\left(4 \tilde{k}^{2} \alpha^{2}\right)} e^{i(-\mathbf{q}+\tilde{k} \mathbf{y} /(\gamma z)) \cdot \mathbf{x}} \\
& \quad \times \exp \left[-\tilde{k}^{2} /\left(2 \gamma^{2}\right) \int_{0}^{z} D_{*}(-s \mathbf{y} / z-\gamma \mathbf{q}(z-s) / \tilde{k}) d s\right] d \mathbf{q} \\
& =\left(\frac{\alpha}{\sqrt{2 \pi}}\right)^{d} \int e^{-|\mathbf{w}|^{2} /\left(4 \alpha^{2}\right)} e^{-|\mathbf{y}-\gamma z \mathbf{w} / \tilde{k}|^{2} /\left(4 \alpha^{2}\right)} e^{i \mathbf{w} \cdot \mathbf{x}} \\
& \quad \times \exp \left[-\tilde{k}^{2} /\left(2 \gamma^{2}\right) \int_{0}^{z} D_{*}(-\mathbf{y}+\gamma \mathbf{w}(z-s) / \tilde{k}) d s\right] d \mathbf{w} .
\end{aligned}
$$

In the regime $\gamma z \ll \tilde{k}$ we define the turbulence-induced (de)coherence length $d_{*}$ as

$$
d_{*}=\sqrt{\frac{\int|\mathbf{y}|^{2} \mathcal{F}\left[T_{2}\right]^{2}(z, 0, \mathbf{y} ; \tilde{k}) d \mathbf{y}}{\int T_{2}^{2}(z, 0, \mathbf{y} ; \tilde{k}) d \mathbf{y}}}
$$

with

$$
T_{2}(z, \mathbf{w}, \mathbf{y} ; \tilde{k})=\exp \left[-\tilde{k}^{2} /\left(2 \gamma^{2}\right) \int_{0}^{z} D_{*}(-\mathbf{y}+\gamma \mathbf{w}(z-s) / \tilde{k}) d s\right] .
$$

Note that $T_{2}(z, 0, \mathbf{y} ; \tilde{k})=\hat{T}(z, \mathbf{y} ; \tilde{k})$. 
4.4.1. Homogeneous medium. Let $R_{0}$ be the coherence function in the homogeneous case. We have

$$
\begin{aligned}
R_{0}(\mathbf{x}, \mathbf{y} ; \tilde{k})= & \left(\frac{2 \alpha^{4}}{1+\gamma^{2} z^{2} / \tilde{k}^{2}}\right)^{d / 2} \exp \left[-\frac{\alpha^{2}|\mathbf{x}|^{2}}{1+\gamma^{2} z^{2} / \tilde{k}^{2}}\right] \\
& \times \exp \left[-\frac{|\mathbf{y}|^{2}}{4 \alpha^{2}\left(1+\gamma^{2} z^{2} / \tilde{k}^{2}\right)}\right] \exp \left[\frac{i \gamma z \mathbf{x} \cdot \mathbf{y}}{\tilde{k}\left(1+\gamma^{2} z^{2} / \tilde{k}^{2}\right)}\right] .
\end{aligned}
$$

The decoherence length $d_{0}$ is then given by

$$
d_{0}=\sqrt{2} \alpha \sqrt{1+\gamma^{2} z^{2} / \tilde{k}^{2}}
$$

4.4.2. Turbulent medium. For $z \gamma \ll \tilde{k} / \eta$ we have the approximation

$$
\begin{aligned}
R_{2}(z, \mathbf{x}, \mathbf{y} ; \tilde{k}) \approx\left(\frac{\alpha}{\sqrt{2 \pi}}\right)^{d} e^{-|\mathbf{y}|^{2} /\left(4 \alpha^{2}\right)} \int e^{-|\mathbf{w}|^{2} /\left(4 \alpha^{2}\right)} e^{i \mathbf{w} \cdot \mathbf{x}} \\
\quad \times \exp \left[-\tilde{k}^{2} /\left(2 \gamma^{2}\right) \int_{0}^{z} C_{*}^{2}|-\mathbf{y}+\gamma \mathbf{w}(z-s) / \tilde{k}|^{2 H_{*}} d s\right] d \mathbf{w} .
\end{aligned}
$$

The above integration is the inverse Fourier transform of a function which, unlike $\exp \left[-|\mathbf{w}|^{2 H_{*}}\right]$ in (4.42), is smooth at $\mathbf{w}=0$ for $\mathbf{y} \neq 0$. Hence its inverse Fourier transform has rapid decay at large $|\mathbf{x}|$. In other words, the two-point correlation decays exponentially fast as the pair of points of a fixed distance move away from the center of the beam, while the intensity of the wave field decays like a power law, as noted in the previous section.

On the other hand, if we let $\mathbf{x}$ be fixed but $\mathbf{y}$ increased, then we can make the further approximation

$$
\begin{aligned}
& R_{2}(z, \mathbf{x}, \mathbf{y} ; \tilde{k}) \\
& \approx \alpha^{d} e^{-|\mathbf{y}|^{2} /\left(4 \alpha^{2}\right)}\left(\int e^{-|\mathbf{w}|^{2} /\left(4 \alpha^{2}\right)} e^{i \mathbf{w} \cdot \mathbf{x}} d \mathbf{w}\right) \exp \left[-\tilde{k}^{2} /\left(2 \gamma^{2}\right) D_{*}(-\mathbf{y}) z\right] \\
& \approx R_{0}(\mathbf{x}, \mathbf{y} ; \tilde{k}) \exp \left[-\tilde{k}^{2} /\left(2 \gamma^{2}\right) D_{*}(-\mathbf{y}) z\right], \quad \gamma \ll 1,
\end{aligned}
$$

and we see that the turbulence-induced (de)coherence length has the asymptotic

$$
\begin{aligned}
& d_{*} \approx \sqrt{\frac{\int|\mathbf{y}|^{2} \hat{T}^{2}(z, 0, \mathbf{y} ; \tilde{k}) d \mathbf{y}}{\int \hat{T}^{2}(z, 0, \mathbf{y} ; \tilde{k}) d \mathbf{y}}}, \quad \gamma \ll 1, \\
& \sim C_{*}^{-\zeta} \tilde{k}^{-\zeta} z^{-\zeta / 2} \gamma^{\zeta} \sim \alpha_{*}, \quad \zeta \in[1,2) .
\end{aligned}
$$

The turbulence-induced (de)coherence length $d_{*}$ represents the intrinsic aperture associated with the turbulent medium, and when the turbulence effect becomes important we have $d_{*} \leq d_{0}$. This happens, for example, when the propagation distance is beyond the boundary layer such that

$$
z \gg \tilde{k}^{-2} \alpha^{-2 H_{*}} \gamma^{2} C_{*}^{-2} .
$$

The forward resolution is $\tilde{s}_{*} \sim s_{*}$ and is related to the speckle size $d_{*}$ as

$$
\tilde{s}_{*} \sim \frac{\gamma z}{\tilde{k} d_{*}} .
$$


We have seen how rough turbulent medium fluctuations affect the wave field and give a wide spreading of the wave energy and a fine scale for the scintillations or the speckle pattern. In the next section we will analyze time reversal of waves. The dual situation can then be observed. For time reversal of waves on a mirror with a given aperture, rough medium fluctuations give a wide apparent mirror and a very fine-scaled time-reversal resolution. We summarize this duality picture in section 6 .

5. Phase-conjugating mirror and superresolution. An important phenomenon in wave propagation in heterogeneous media is related to time reversal of the wave field. In a generic time-reversal example the wave received by an active transducer or antenna (receiver-emitter) array is recorded and then reemitted into the medium time reversed; that is, the tails of the recorded signals are sent first. The time-reversal procedure is equivalent to phase conjugation on the spatial component of the time-harmonic wave field which, in the parabolic approximation, is the same as changing the sign of the wave number $\tilde{k}$. Phase conjugation is an essential element in holography. Phase conjugation can be produced from a holographic procedure of four-wave mixing in a photorefractive crystal [32].

The phenomenon is related to the time reversibility of the wave equation; if we capture, time reverse, and reemit a sufficient part of the wave field, the reemitted wave will approximately refocus on the target [18]. The surprising and important fact is that the focusing resolution typically will be enhanced rather than hampered by heterogeneity or "randomness" in the medium. The effect has numerous applications. In the case of ultrasound, this process can be iterated to pinpoint the wave beam to destroy kidney stones, detect defects in materials, and communicate with submarines. In the case of electromagnetic waves this effect holds the potential of increasing imaging resolution and channel capacity. The phenomenon has been studied in the literature, both from the experimental and theoretical points of view $[2,5,6,4,11,18,19,20,21,28,31]$. A cartoon of a time-reversal experiment is given in Figure 5.1.

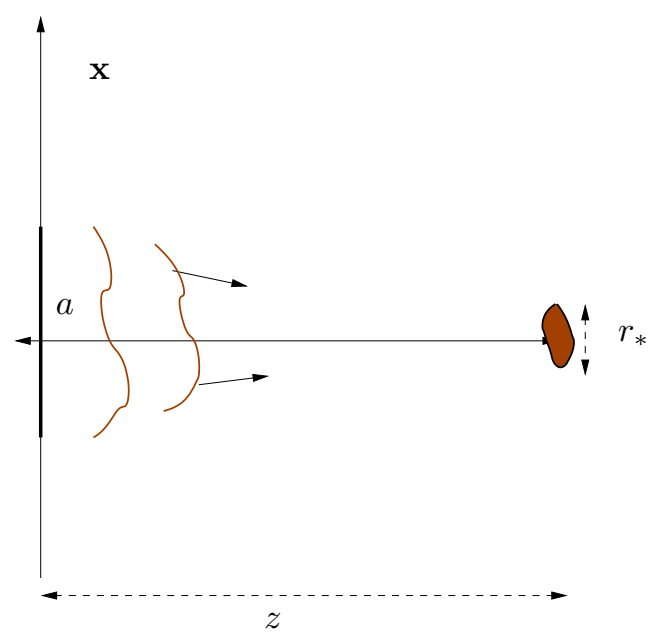

FIG. 5.1. The time-reversal procedure. A source with central wavelength $\lambda_{0}$ emits a pulse. The transmitted field is recorded, stored, and time reversed at the mirror of size a at a distance $z$ away and then sent back toward the source point. There it refocuses on the spot size, $r_{*}$, described by (5.12) when the medium is homogeneous. Medium heterogeneity typically enhances the refocusing resolution. 
A crucial aspect of this effect is the enhanced performance of time-reversal resolution by heterogeneity or medium roughness. Previous theoretical studies typically assume that the medium is smooth in the sense of being differentiable with respect to the lateral coordinates; see [35] and [36] for examples. There, scaling regimes associated with a robust and strong refocusing effect were identified by asymptotic analysis, and the refocusing effect was precisely quantified.

Fractal-like self-similar media have much "rougher" variations and occur naturally in wave propagation in the atmosphere and geophysical setting. They are the main focus of the present study.

5.1. Time-reversed field. As in (3.4) we nondimensionalize the coordinates. Let the phase-conjugated mirror be located on the plane $z=0$ and the source at the parallel plane a $z$-distance away with an aperture $A$. The aperture function of the mirror is, in the simplest form, the indicator function $\chi_{A}$ of the set $A$ representing the physical boundary of the mirror.

Interestingly, the phase-conjugated, back-propagated wave field can be related to the Wigner distribution as follows. Let $G_{H}(0, \mathbf{x}, z, \mathbf{y})$ be the Green's function, with the point source located at $(z, \mathbf{y})$, for the reduced wave (Helmholtz) equation for which the Schrödinger-Itô equation is an approximation. By the self-adjointness of the Helmholtz equation, $G_{H}$ satisfies the symmetry property

$$
G_{H}(0, \mathbf{x}, z, \mathbf{y})=G_{H}(z, \mathbf{y}, 0, \mathbf{x}) .
$$

The wave field $\Psi_{m}$ received at the mirror is given by

$$
\begin{aligned}
\Psi_{m}\left(z, \mathbf{x}_{m}\right) & =\chi_{A}\left(\mathbf{x}_{m}\right) \int G_{H}\left(0, \mathbf{x}_{m}, z, \mathbf{x}_{s}\right) \Psi_{0}\left(\mathbf{x}_{s}\right) d \mathbf{x}_{s} \\
& =\chi_{A}\left(\mathbf{x}_{m}\right) \int G_{H}\left(z, \mathbf{x}_{s}, 0, \mathbf{x}_{m}\right) \Psi_{0}\left(\mathbf{x}_{s}\right) d \mathbf{x}_{s} .
\end{aligned}
$$

After phase conjugation and back-propagation we have at the source plane the wave field

$$
\Psi^{B}(z, \mathbf{x} ; \tilde{k})=\int G_{H}\left(z, \mathbf{x}, 0, \mathbf{x}_{m}\right) \overline{G_{H}\left(z, \mathbf{x}_{s}, 0, \mathbf{x}_{m}\right)} \chi_{A}\left(\mathbf{x}_{m}\right) \overline{\Psi_{0}\left(\mathbf{x}_{s}\right)} d \mathbf{x}_{m} d \mathbf{x}_{s} .
$$

In the parabolic and white-noise approximations the Green's function $G_{H}(z, \mathbf{x}, 0, \mathbf{y})$ is approximated by $e^{i \tilde{k} z} G(z, \mathbf{x}, \mathbf{y})$, where $G_{S}(z, \mathbf{x}, \mathbf{y})$ is the propagator of the Schrödinger-Itô equation (3.15). Making the approximation in the above expression for the back-propagated field we obtain

$$
\begin{aligned}
\Psi^{B}(z, \mathbf{x} ; \tilde{k}) & =\int G_{S}\left(z, \mathbf{x}, \mathbf{x}_{m}\right) \overline{G_{S}\left(z, \mathbf{x}_{s}, \mathbf{x}_{m}\right) \Psi_{0}\left(\mathbf{x}_{s}\right)} \chi_{A}\left(\mathbf{x}_{m}\right) d \mathbf{x}_{m} d \mathbf{x}_{s} \\
& =\int e^{i \mathbf{p} \cdot\left(\mathbf{x}-\mathbf{x}_{s}\right) / \gamma} W\left(z, \frac{\mathbf{x}+\mathbf{x}_{s}}{2}, \mathbf{p}\right) \overline{\Psi_{0}\left(\mathbf{x}_{s}\right)} d \mathbf{p} d \mathbf{x}_{s},
\end{aligned}
$$

where the Wigner distribution $W$ is given by

$$
\begin{aligned}
& W(z, \mathbf{x}, \mathbf{p}) \\
& =\frac{1}{(2 \pi)^{d}} \int e^{-i \mathbf{p} \cdot \mathbf{y}} G_{S}\left(z, \mathbf{x}+\gamma \mathbf{y} / 2, \mathbf{x}_{m}\right) \overline{G_{S}\left(z, \mathbf{x}-\gamma \mathbf{y} / 2, \mathbf{x}_{m}\right)} \chi_{A}\left(\mathbf{x}_{m}\right) d \mathbf{y} d \mathbf{x}_{m}
\end{aligned}
$$

This is a mixed-state type of Wigner distribution (3.26). In general, the integral in (5.1) should be interpreted in the distributional sense. 
The Wigner distribution in (5.2) has the initial condition $W$,

$$
W(0, \mathbf{x}, \mathbf{p})=\frac{\chi_{A}(\mathbf{x})}{\gamma^{d}(2 \pi)^{d}},
$$

which is an $L^{\infty}\left(\mathbb{R}^{2 d}\right)$-function and should be treated as a generalized function on $\mathbb{R}^{2 d}$. Indeed, for any $\theta \in C_{c}^{\infty}\left(\mathbb{R}^{d}\right)$ we have

$$
\left\langle\Psi^{B}, \theta\right\rangle=\iint W(z, \mathbf{r}, \mathbf{p}) \Theta(\mathbf{r}, \mathbf{p}) d \mathbf{y} d \mathbf{p},
$$

where the function $\Theta$ is defined as

$$
\Theta(\mathbf{r}, \mathbf{p})=2^{d} \int \theta\left(\mathbf{r}^{\prime}\right) e^{i 2 \mathbf{p} \cdot\left(\mathbf{r}^{\prime}-\mathbf{r}\right) / \gamma} \overline{\Psi_{0}\left(2 \mathbf{r}-\mathbf{r}^{\prime}\right)} d \mathbf{r}^{\prime} .
$$

If, for instance, $\Psi_{0} \in C_{c}^{\infty}\left(\mathbb{R}^{d}\right)$, then it is easy to see $\Theta(\mathbf{y}, \mathbf{p})$ is compactly supported in $\mathbf{y} \in \mathbb{R}^{d}$ and decays rapidly (faster than any power) in $\mathbf{p} \in \mathbb{R}^{d}$. Therefore the mixedstate Wigner distribution given by (5.2) can be treated as a tempered distribution. As a result we can always approximate to arbitrary accuracy the distributional initial data such as (5.3) by square-integrable initial data whose evolution is in turn approximately described by the Wigner-Itô equation (3.23).

Following (5.1) we can write the coherent field $\bar{\Psi}^{B} \equiv \mathbb{E}\left[\Psi^{B}\right]$ in terms of $W^{(1)}$ as

$$
\bar{\Psi}^{B}(z, \mathbf{x} ; \tilde{k})=\int e^{i \mathbf{p} \cdot\left(\mathbf{x}-\mathbf{x}_{s}\right) / \gamma} W^{(1)}\left(z, \frac{\mathbf{x}+\mathbf{x}_{s}}{2}, \mathbf{p}\right) \overline{\Psi_{0}\left(\mathbf{x}_{s}\right)} d \mathbf{p} d \mathbf{x}_{s} .
$$

In contrast, an ordinary mirror would result in the following expression for the backpropagated wave field $\Psi_{b}$ :

$$
\Psi_{b}(z, \mathbf{x})=\int G_{S}\left(z, \mathbf{x}, \mathbf{x}_{m}\right) G_{S}\left(z, \mathbf{x}_{s}, \mathbf{x}_{m}\right) \Psi_{0}\left(\mathbf{x}_{s}\right) \chi_{A}\left(\mathbf{x}_{m}\right) d \mathbf{x}_{m} d \mathbf{x}_{s}
$$

which upon averaging becomes

$$
\bar{\Psi}_{b}(z, \mathbf{x})=\int F^{(2)}\left(z, \mathbf{x}, \mathbf{x}_{s}\right) \Psi_{0}\left(\mathbf{x}_{s}\right) d \mathbf{x}_{s},
$$

where

$$
F^{(2)}\left(z, \mathbf{x}, \mathbf{x}_{s}\right)=\int G_{S}^{(2)}\left(z, \mathbf{x}, \mathbf{x}_{s} ; \mathbf{x}_{m}, \mathbf{x}_{m}\right) \chi_{A}\left(\mathbf{x}_{m}\right) d \mathbf{x}_{m}
$$

is the solution of (3.17) for $n=2$ with the initial data

$$
F_{0}^{(2)}\left(\mathbf{x}, \mathbf{x}_{s}\right)=\delta\left(\mathbf{x}-\mathbf{x}_{s}\right) \chi_{A}(\mathbf{x})
$$

The problem for $F^{(2)}$ does not lead to a simple solution, as is the case for $W^{(1)}$.

Recall that the governing equation for $W^{(1)}$ is given by the averaged WignerItô equation (4.12) and the associated Green's function has the form given in (4.13). Thus, the explicit expression for $W^{(1)}$ taking the initial data into account is in this case

$$
\begin{aligned}
W^{(1)}(z, \mathbf{x}, \mathbf{p})= & \frac{1}{(2 \pi \gamma) d} \int G_{W}^{(1)}(z, \mathbf{x}, \mathbf{p} ; \overline{\mathbf{x}}, \overline{\mathbf{p}}) \chi_{A}(\overline{\mathbf{x}}) d \overline{\mathbf{x}} d \overline{\mathbf{p}} \\
= & \frac{1}{\gamma^{d}(2 \pi)^{2 d}} \int \chi_{A}(\overline{\mathbf{x}}) \exp [i(\mathbf{w} \cdot(\mathbf{x}-\overline{\mathbf{x}})-z \mathbf{w} \cdot \mathbf{p} / \tilde{k})] \\
& \quad \times \exp \left[-\tilde{k}^{2} /\left(2 \gamma^{2}\right) \int_{0}^{z} D_{*}(-\gamma \mathbf{w} s / \tilde{k}) d s\right] d \mathbf{w} d \overline{\mathbf{x}}
\end{aligned}
$$


This then gives an expression for the quantity of interest, the mean time-reversed and back-propagated field:

$$
\begin{aligned}
\bar{\Psi}^{B}(z, \mathbf{x} ; \tilde{k})= & \frac{1}{\gamma^{d}(2 \pi)^{d}} \int \overline{\hat{\Psi}_{0}\left(\frac{\mathbf{p}}{\gamma}\right)} \chi_{A}(\overline{\mathbf{x}}) e^{i\left(\mathbf{p} \cdot \mathbf{x} / \gamma+\mathbf{w} \cdot \mathbf{x}-\mathbf{w} \cdot \overline{\mathbf{x}}-z \mathbf{w} \cdot \mathbf{p} / \tilde{k}-\gamma z|\mathbf{w}|^{2} / 2 \tilde{k}\right)} \\
& \times \exp \left[-\tilde{k}^{2} /\left(2 \gamma^{2}\right) \int_{0}^{z} D_{*}(-s \gamma \mathbf{w} / \tilde{k}) d s\right] d \overline{\mathbf{x}} d \mathbf{p} d \mathbf{w} \\
= & \frac{1}{(2 \pi)^{d}} \int \overline{\hat{\Psi}_{0}(\mathbf{q})} \chi_{A}(\overline{\mathbf{x}}) e^{i\left(\mathbf{q} \cdot \mathbf{x}+\mathbf{w} \cdot \mathbf{x}-\mathbf{w} \cdot \overline{\mathbf{x}}-\gamma z \mathbf{w} \cdot \mathbf{q} / \tilde{k}-\gamma z|\mathbf{w}|^{2} / 2 \tilde{k}\right)} \\
& \times \exp \left[-\tilde{k}^{2} /\left(2 \gamma^{2}\right) \int_{0}^{z} D_{*}(-s \gamma \mathbf{w} / \tilde{k}) d s\right] d \overline{\mathbf{x}} d \mathbf{q} d \mathbf{w} .
\end{aligned}
$$

We introduce the time-reversal point-spread function $\mathcal{P}_{\text {tr }}$ by substituting the Diracdelta function for $\Psi_{0}$ in the above expression:

$$
\begin{aligned}
\mathcal{P}_{\operatorname{tr}}(z, \mathbf{x} ; \tilde{k})= & \frac{1}{(2 \pi)^{d}} \int \hat{\chi}_{A}(\mathbf{w}) e^{i[\mathbf{q} \cdot(\mathbf{x}-\gamma z \mathbf{w} / \tilde{k})+\mathbf{w} \cdot(\mathbf{x}-\gamma z \mathbf{w} / 2 \tilde{k})]} \\
& \times \exp \left[-\tilde{k}^{2} /\left(2 \gamma^{2}\right) \int_{0}^{z} D_{*}(-s \gamma \mathbf{w} / \tilde{k}) d s\right] d \mathbf{q} d \mathbf{w} \\
= & \left(\frac{\tilde{k}}{\gamma z}\right)^{d} \hat{\chi}_{A}\left(\frac{\tilde{k} \mathbf{x}}{\gamma z}\right) \exp \left[i \tilde{k}|\mathbf{x}|^{2} /(2 \gamma z)\right] \exp \left[-\tilde{k}^{2} /\left(2 \gamma^{2}\right) z \int_{0}^{1} D_{*}(-s \mathbf{x}) d s\right] .
\end{aligned}
$$

The mean refocused wave field has a spatial support that is determined by the resolution function

$$
\tilde{\mathcal{P}}(z, \mathbf{x} ; \tilde{k}) \equiv\left(\frac{\tilde{k}}{\gamma z}\right)^{d} \hat{\chi}_{A}\left(\frac{\tilde{k} \mathbf{x}}{\gamma z}\right) \exp \left[-\tilde{k}^{2} /\left(2 \gamma^{2}\right) z \int_{0}^{1} D_{*}(-s \mathbf{x}) d s\right] .
$$

It is clear from (5.8) that the effect of the random media is the damping of the homogeneous resolution function

$$
\tilde{\mathcal{P}}_{0}(z, \mathbf{x} ; \tilde{k})=\left(\frac{\tilde{k}}{\gamma z}\right)^{d} \hat{\chi}_{A}\left(\frac{\tilde{k} \mathbf{x}}{\gamma z}\right)
$$

by the phase factor

$$
T_{\mathrm{tr}} \equiv \exp \left[-\tilde{k}^{2} /\left(2 \gamma^{2}\right) z \int_{0}^{1} D_{*}(-s \mathbf{x}) d s\right] .
$$

The homogeneous resolution function (5.9) is analogous to the focusing of uniform light through the aperture stop $A$ by a lens on its focal plane. The lensing effect results, of course, from phase conjugation. The homogeneous resolution function (5.9) converges in the sense of distribution to the Dirac-delta function in the limit of infinite aperture $A \rightarrow \mathbb{R}^{d}$. To further analyze the differences between the resolution functions of homogeneous and random media, we will now set $A$ to be a circular aperture of diameter $a$. 
5.1.1. Homogeneous medium. For a circular aperture we have the well-known formula [7]

$$
\tilde{\mathcal{P}}_{0}=\frac{a \tilde{k}}{4 \pi \gamma z|\mathbf{x}|} J_{1}\left(\frac{\tilde{k}|\mathbf{x}| a}{2 \gamma z}\right)
$$

where $J_{1}$ is the first order Bessel function given by

$$
J_{1}(w)=\frac{1}{2 \pi} \int_{0}^{2 \pi} e^{i \phi+i w \sin \phi} d \phi
$$

The Rayleigh resolution is then simply the distance $\rho_{0}$ to the first zero of $\tilde{\mathcal{P}}_{0}(5.11)$ or the Airy spot size:

$$
\rho_{0} \approx 1.22 \gamma \frac{\tilde{\lambda} z}{a}, \quad \tilde{\lambda}=\frac{2 \pi}{\tilde{k}},
$$

which is analogous to (4.54) and (4.62). One sees that the resolution is proportional to $\gamma$ and the distance to the mirror and inversely proportional to the aperture $a$. The Rayleigh criterion is valid only for illumination by an incoherent light source [7].

Note that (5.12) is not in the standard form since we have rescaled and nondimensionalized the coordinates (3.4) such that $\tilde{k} \approx 1$ and the true dependence of $\rho_{0}$ on the wavelength is hidden in $\gamma$.

5.2. Turbulent medium: Superresolution and contrast enhancement. In the presence of random fluctuations we have

$$
\tilde{\mathcal{P}}(z, \mathbf{x} ; \tilde{k}) \equiv \frac{a \tilde{k}}{4 \pi \gamma z|\mathbf{x}|} J_{1}\left(\frac{\tilde{k}|\mathbf{x}| a}{2 \gamma z}\right) \exp \left[-\tilde{k}^{2} z \int_{0}^{1} D_{*}(-s \mathbf{x}) d s /\left(2 \gamma^{2}\right)\right] .
$$

As $\gamma$ decreases, the random phase factor (5.10) with a rough structure function may contain a smaller scale than (5.12) resulting in a turbulence-induced time-reversal aperture $\alpha_{\mathrm{tr}}$ which is independent of the entrance aperture $a$.

For the power-law structure function given in (4.15) we obtain

$$
\begin{aligned}
& \tilde{\mathcal{P}}(z, \mathbf{x} ; \tilde{k}) \\
& \approx \frac{a \tilde{k}}{4 \pi \gamma z|\mathbf{x}|} J_{1}\left(\frac{\tilde{k}|\mathbf{x}| a}{2 \gamma z}\right) \exp \left[-C_{*}^{2} \tilde{k}^{2} z|\mathbf{x}|^{2 H_{*}} \gamma^{-2} /\left(4 H_{*}+2\right)\right], \quad|\mathbf{x}| \ll 1,
\end{aligned}
$$

with $H_{*}$ given by (4.16). The function

$$
T_{\mathrm{tr}}(z, \mathbf{x})=\exp \left[-C_{*}^{2} \tilde{k}^{2} z|\mathbf{x}|^{2 H_{*}} \gamma^{-2} /\left(4 H_{*}+2\right)\right]
$$

in (5.14) determines the time-reversal resolution when

$$
s_{*} \sim \tilde{s}_{*} \gg a
$$

In this case the turbulence-induced time-reversal resolution defined as

$$
\rho_{\mathrm{tr}}=\sqrt{\frac{\int|\mathbf{x}|^{2} T_{\mathrm{tr}}^{2\left(2 H_{*}+1\right)}(z, \mathbf{x} ; \tilde{k}) d \mathbf{x}}{\int T_{\mathrm{tr}}^{2\left(2 H_{*}+1\right)}(z, \mathbf{x} ; \tilde{k}) d \mathbf{x}}}
$$


has the asymptotic for $z^{-1 / 2} \gamma \ll 1$,

$$
\rho_{\mathrm{tr}} \sim C_{*}^{-\zeta} \tilde{k}^{-\zeta} z^{-\zeta / 2} \gamma^{\zeta} \sim d_{*} \sim \hat{s}_{*}^{-1}, \quad \zeta=1 / H_{*} \in[1,2),
$$

corresponding to the turbulence-induced time-reversal aperture

$$
\alpha_{\mathrm{tr}} \equiv \frac{\gamma z}{\tilde{k} \rho_{\mathrm{tr}}} \sim C_{*}^{\zeta} \tilde{k}^{\zeta-1} z^{1+\zeta / 2} \gamma^{1-\zeta} \sim s_{*} \gg a
$$

It is worthwhile to mention that when the formula (5.16) is valid the time-reversal resolution improves as the propagation distance $z$ and the random fluctuation (measured by $C_{*}$ ) of the medium increase and as the Fresnel number $\gamma$ decreases.

The case $\zeta=1$ corresponds to a smooth white-noise medium, while the case $\zeta=2$ corresponds to a rough white-noise medium as before (cf. section 4.1). The rougher the medium, the smaller the exponent $H_{*}$, and so is the resolution scale (5.17). In the case of a Gaussian white-noise model for the Liouville equation with a smooth potential (i.e., $\zeta=1$ ), the turbulence-induced time-reversal aperture has a similar dependence on $z$ (i.e., $z^{3 / 2}$ ) [36].

The definition (5.18) of the turbulence-induced time-reversal aperture $\alpha_{\mathrm{tr}}$ is completely analogous to the definition (4.54) of the turbulence-induced entrance aperture $\alpha_{*}$. By definition the turbulence-induced aperture is the largest aperture for which the diffraction-limited resolution is not affected by the random medium. Even when the size of the phase-conjugating mirror is bigger than $\alpha_{\text {tr }}$ given by (5.18), and hence a smaller diffraction-limited resolution than (5.17), the presence of randomness has the advantage of enhancing the contrast between the refocused spot and the surrounding wave field due to the fast decay of the stretched exponential function in (5.14). Physically speaking, it is no coincidence that $\alpha_{\operatorname{tr}} \sim \tilde{s}_{*}$ once the duality between $\mathbb{E}\left[\Psi^{B}\right]$ and $\hat{M}_{2}$ is established (see the next section).

At first the aperture-independent resolution formula (5.17) seems counterintuitive. On the other hand, it is hardly surprising that the scale of refocusing of the coherent back-propagated field has the same order of magnitude as the decoherence length (4.60) of the transmitted beam. As a consequence, the coherent back-propagated wave field behaves analogously to incoherent light wave, and the time-reversal Airy spot size $\rho_{\text {tr }}$ gives the true resolution of distinguishing two points. As noted in the previous section, $\rho_{\operatorname{tr}}(5.17)$ is $o(1)$, as long as the phase-conjugating mirror is placed beyond the boundary layer (4.61). However, the question of whether (5.17) remains valid for the time-reversal focal spot size in a given realization of the random medium must be answered by considering the higher moments of the wave field, the so-called scintillation theory which we will analyze in a separate paper. Here we briefly remark that at least for a broad beam $\alpha \gg 1$ (5.17) indeed describes the time-reversal refocusing scale for almost all realizations of the medium. Indeed, the focal spot itself is statistically stable. This is the self-averaging effect observed in [5] and further analyzed in [36]. See [14] for a comprehensive analysis of the self-averaging effect in various scaling limits.

Another aspect of the superresolution is the enhanced contrast or band-width of the time-reversal focal spot due to the much faster decay of the stretched exponential function in (5.14) than $J_{1}$ at large offset $\mathbf{x}$. For instance, we can define the time-reversal contrast or band-width $\kappa_{\mathrm{tr}}$ as the ratio of the central, peak intensity $\left|\mathcal{P}_{\operatorname{tr}}(z, 0 ; \tilde{k})\right|^{2}$ to the total back-propagated energy which has the approximation when 
the effect of the random medium dominates:

$$
\begin{aligned}
\kappa_{\mathrm{tr}} & =\frac{\left|\mathcal{P}_{\operatorname{tr}}(z, 0 ; \tilde{k})\right|^{2}}{\int\left|\mathcal{P}_{\operatorname{tr}}(z, \mathbf{x} ; \tilde{k})\right|^{2} d \mathbf{x}} \\
& \sim\left(\int T_{\mathrm{tr}}^{2}(z, \mathbf{x}) d \mathbf{x}\right)^{-1} \\
& =\left(\tilde{k}^{-\zeta} C_{*}^{-\zeta} \gamma^{\zeta} z^{-\zeta / 2}\right)^{-d}\left(\int \exp \left[-|\mathbf{x}|^{2 H_{*}} /\left(2 H_{*}+1\right)\right] d \mathbf{x}\right)^{-1} \\
& \sim \rho_{\mathrm{tr}}^{-d}
\end{aligned}
$$

which increases as $\gamma$ and $H$ decrease and as $C_{*}$ and $z$ increase, and is much better than the time-reversal contrast in the homogeneous medium. This dependence on the parameters $\gamma, H, C_{*}, z$ is opposite to that of the forward contrasts as given in (4.52).

The price for the enhanced contrast is, however, the significant reduction of the energy of the focal spot by the factor $\kappa_{\mathrm{tr}}^{-1} \sim \rho_{\mathrm{tr}}^{d}$ due to the reduction of the side lobes, although the intensity at the center of the spot $\mathbf{x}=0$ remains the same as the homogeneous case (Strehl ratio $\approx 1$ ). The loss of refocused energy cannot always be completely compensated by enlarging the aperture or decreasing the Fresnel number $\gamma$. For example, to refocus the same amount of wave energy as in the homogeneous case, we need to increase $a /(z \gamma)$ by a factor of $\rho_{\mathrm{tr}}^{-2}$, but then the Rayleigh resolution would have been much better than $\rho_{\text {tr }}$ anyway.

5.3. Refocusing in time. The setting is the same as before: the source is located at $(z, 0)$, while the phase-conjugating mirror is at $(0,0)$; see Figure 5.1.

By decomposing the initial pulse $u_{0}(\tau, \mathbf{x})$ into its various frequency components $\Psi_{0}(\mathbf{x} ; \tilde{k})$ as

$$
u_{0}(\tau, \mathbf{x})=\int \Psi_{0}(\mathbf{x} ; \tilde{k}) e^{-i \tilde{k}(\tau+\nu z)} d \tilde{k}, \quad \nu=k_{0} L_{z}, \quad \tau=c_{0} k_{0} t,
$$

we can apply the time-reversal procedure frequency by frequency and then synthesize them to obtain the refocused wave field

$$
\mathbb{E}\left[u^{B}\right](\tau, \mathbf{x})=\int \overline{u_{0}(t, \mathbf{y})} \mathcal{Q}_{\operatorname{tr}}(\tau+t, \mathbf{x}, \mathbf{y}) d \mathbf{y} d t
$$

where the space-time time-reversal point-spread function $\mathcal{Q}_{\operatorname{tr}}(\tau, \mathbf{x}, \mathbf{y})$ is given by

$$
\begin{aligned}
& \mathcal{Q}_{\operatorname{tr}}(\tau, \mathbf{x}, \mathbf{y})=\frac{1}{2 \pi}\left(\frac{1}{\gamma z}\right)^{2} \int e^{-i \tilde{k} t} e^{\frac{i \tilde{k}|\mathbf{x}|^{2}}{2 \gamma z}} e^{-\frac{i \tilde{k}|\mathbf{y}|^{2}}{2 \gamma z}} \tilde{k}^{2} \hat{\chi}_{A}\left(\frac{\tilde{k}(\mathbf{x}+\mathbf{y})}{\gamma z}\right) \\
& \times \exp \left[-\frac{\tilde{k}^{2} z}{2 \gamma^{2}} \int_{0}^{1} D_{*}(s(\mathbf{x}-\mathbf{y})) d s\right] d \tilde{k} \\
& =\frac{1}{\left(2 \pi \sigma_{\mathrm{tr}}(\mathbf{x}-\mathbf{y})\right)^{2}} \int \mathcal{N}_{\sigma_{\mathrm{tr}}(\mathbf{x}-\mathbf{y})}\left(\tau-t-\frac{|\mathbf{x}|^{2}-|\mathbf{y}|^{2}}{2 \gamma z}\right) f_{A}(t,|\mathbf{x}+\mathbf{y}|) d t,
\end{aligned}
$$

where $\mathcal{N}_{\sigma_{\text {tr }}(\mathbf{r})}$ is the normal distribution with the variance

$$
\sigma_{\operatorname{tr}}(\mathbf{r})=\frac{1}{\gamma} \sqrt{z \int_{0}^{1} D_{*}(-s \mathbf{r}) d s}
$$


and the function $f_{A}$ is given by

$$
f_{A}(t, r)=\frac{\gamma z}{\pi a r^{3}} f\left(\frac{2 \gamma z t}{a r}\right), \quad f(s)=\int w J_{1}(w) e^{-i s w} d w .
$$

We call $\sigma_{\text {tr }}$ the turbulence-induced temporal spread for time reversal. In view of (A.10) we see that the scale associated with $\sigma_{\text {tr }}$ is proportional to the turbulenceinduced cross-range resolution $\rho_{\operatorname{tr}}$ as given by (5.16). This is contrary to the offsetindependent temporal spread $\sigma$ of the forward propagating mean field as given by (4.6). We note that in the case of longitudinally long-range correlated fractal media the temporal pulse-shaping function $\mathcal{N}_{\sigma_{\text {tr }}}$ may be a non-Gaussian function [16].

Consider the simplest case of a point source

$$
u_{0}(\tau, \mathbf{x})=\delta(\mathbf{x}) \int \hat{g}(\tilde{k}) e^{-i \tilde{k}(\tau+\nu z)} d \tilde{k} .
$$

Then we have the back-propagated pulse

$$
\mathbb{E}\left[u^{B}\right](\tau, \mathbf{x})=\int \bar{g}(t) f_{A}\left(t^{\prime},|\mathbf{x}|\right) \mathcal{N}_{\sigma_{\operatorname{tr}}(\mathbf{x})}\left(\tau+t-t^{\prime}-\frac{|\mathbf{x}|^{2}}{2 \gamma z}\right) d t^{\prime} d t
$$

The temporal spread due to propagation is given by

$$
\frac{a|\mathbf{x}|}{\gamma z}+\sigma_{\mathrm{tr}}(\mathbf{x})
$$

which vanishes at $|\mathbf{x}|=0$ and increases with $|\mathbf{x}|$. In the turbulence-dominated regime $\alpha_{\text {tr }} \gg a$ the second term $\sigma_{\text {tr }}$ increases faster than the first term. On one hand, the rapid increase of the temporal spread with the offset is the mechanism for sharp refocusing in space time for a point source; on the other hand, when the pulse source has a wide lateral support the temporal refocusing may deteriorate due to the interference of the temporal spread from different source points.

6. Duality between transmission and time reversal. In this section we would like to formulate a duality relation between $\hat{M}_{2}$ and $R_{2}$ and between $\hat{M}_{2}$ and $\tilde{\mathcal{P}}$ to explain the observed reciprocity between $\hat{s}_{*}$ and $d_{*}$ and between $\hat{s}_{*}$ and $\rho_{\mathrm{tr}}$, respectively. For simplicity we assume that $\hat{M}_{2}$ is centered, i.e., $\overline{\mathbf{p}}=0$.

The duality is based on the straightforward scaling relation

$$
\frac{\||\mathbf{x}| f\|_{2}}{\|f\|_{2}} \times \frac{\||\mathbf{p}| \mathcal{F}[f]\|_{2}}{\|\mathcal{F}[f]\|_{2}}=\frac{\left\||\mathbf{x}| f_{\lambda}\right\|_{2}}{\left\|f_{\lambda}\right\|_{2}} \times \frac{\left\||\mathbf{p}| \mathcal{F}\left[f_{\lambda}\right]\right\|_{2}}{\left\|\mathcal{F}\left[f_{\lambda}\right]\right\|_{2}} \quad \forall \lambda>0
$$

between any tempered function $f$ and its rescaled version $f_{\lambda}(\mathbf{x})=f(\lambda \mathbf{x})$, where $\mathcal{F}[f]$ denotes the Fourier transform of $f$ as before.

First consider the function

$$
f(\mathbf{x})=\exp \left[-|\mathbf{x}|^{2 H_{*}} / 2\right]
$$

then

$$
\hat{T}(z, \mathbf{x}) \approx \exp \left[-C_{*}^{2} \tilde{k}^{2} z|\mathbf{x}|^{2 H_{*}} /\left(2 \gamma^{2}\right)\right]
$$

can be written as

$$
\hat{T}(z, \mathbf{x}) \approx f_{\lambda}(\mathbf{x})
$$


with

$$
\lambda=C_{*}^{\zeta} \tilde{k}^{\zeta} z^{\zeta / 2} \gamma^{-\zeta}
$$

Now applying the scaling relation above to $f$ given in (6.1) we obtain

$$
\frac{\||\mathbf{x}| \hat{T}\|_{2}}{\|\hat{T}\|_{2}} \times \frac{\||\mathbf{p}| \mathcal{F}[\hat{T}]\|_{2}}{\|\mathcal{F}[\hat{T}]\|_{2}}=\frac{\||\mathbf{x}| f\|_{2}}{\|f\|_{2}} \times \frac{\||\mathbf{p}| \mathcal{F}[f]\|_{2}}{\|\mathcal{F}[f]\|_{2}} \equiv u_{H}
$$

whose right-hand side is independent of $\lambda$ and $z$ and varies mildly with the medium roughness. In view of (4.60) and (4.46) this explains the reciprocity

$$
d_{*} \hat{s}_{*} \approx u_{H}
$$

cf. (4.60) and (4.49). The constant $u_{H}$ has a universal lower bound,

$$
u_{H} \geq \sqrt{\frac{d}{2(2 \pi)^{d}}},
$$

which is achieved for the Gaussian function with $H_{*}=1$ corresponding to $H \geq 1 / 2$ or persistently correlated media.

For the reciprocity of $\hat{s}_{*}$ and $\rho_{\mathrm{tr}}$ we consider next

$$
T_{\mathrm{tr}}(z, \mathbf{x}) \approx \exp \left[-C_{*}^{2} \tilde{k}^{2} z|\mathbf{x}|^{2 H_{*}} \gamma^{-2} /\left(4 H_{*}+2\right)\right]
$$

which can be written as

$$
T_{\operatorname{tr}}(z, \mathbf{x}) \approx \hat{T}(z, \mathbf{x})^{1 /\left(2 H_{*}+1\right)}
$$

We find therefore that

$$
T_{\mathrm{tr}}^{2\left(2 H_{*}+1\right)}(z, \mathbf{x} ; \tilde{k}) / \int T_{\mathrm{tr}}^{2\left(2 H_{*}+1\right)}(z, \mathbf{x} ; \tilde{k}) d \mathbf{x} \approx \hat{T}^{2}(z, \mathbf{x}) / \int T^{2}(z, \mathbf{x}) d \mathbf{x} .
$$

In view of the definition of $\rho_{\operatorname{tr}}$ in (5.16) the duality relation (6.2) again implies the reciprocity of $\rho_{\operatorname{tr}}$ and $\hat{s}_{*}$ :

$$
\rho_{\mathrm{tr}} \hat{s}_{*} \approx u_{H}
$$

In summary we have analyzed the relations among five length scales associated with the effect of the turbulent medium:

- Spread in wavevector, $\hat{s}_{*}$, of the transmitted wave energy.

- Coherence length, $d_{*}$, of the transmitted wave field.

- Entrance aperture, $\alpha_{*}$, of the "lensing" of the turbulent medium.

- Time-reversal resolution, $\rho_{\mathrm{tr}}$, which is the time-reversed focal spot size.

- Time-reversal aperture, $\alpha_{\mathrm{tr}}$, aperture of the phase-conjugating mirror which can reproduce the time-reversal resolution in the absence of the turbulence. 
First, we have the duality relations

$$
\alpha_{*}=\frac{1}{\hat{s}_{*}}
$$

and

$$
\rho_{\mathrm{tr}} \approx d_{*} \approx \frac{u_{H}}{\hat{s}_{*}}
$$

where $u_{H}$ is a constant depending only on $H$. Second, we have

$$
\alpha_{\mathrm{tr}} \approx \frac{\gamma z}{\tilde{k} u_{H}} \hat{s}_{*}=\frac{\tilde{s}_{*}}{u_{H}} .
$$

This duality picture is illustrated in Figure 6.1.

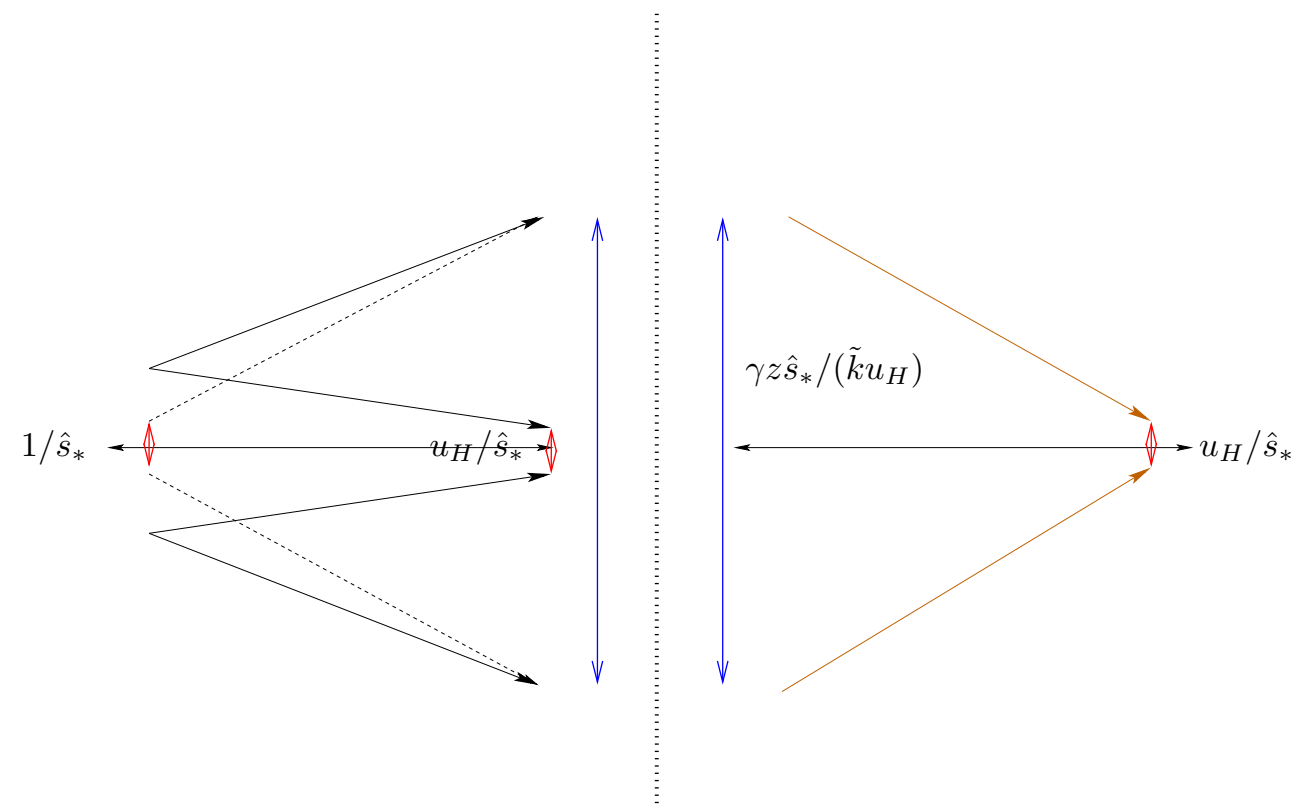

FIG. 6.1. The left and right half figures indicate the turbulence-induced scales for the transmitted field and the time-reversed and back-propagated field, respectively. The arrows indicate the direction of propagation. For the transmitted field the solid vertical line to the left indicates the spread in spatial frequency $\tilde{s}_{*}=\gamma z \hat{s}_{*} /\left(\tilde{k} u_{H}\right)$. The entrance aperture $\alpha_{*}=1 / \hat{s}_{*}$ is shown to the far left, and the coherence length of the transmitted field $d_{*} \approx u_{H} / \hat{s}_{*}$ is marked at the center of the plot. The time-reversal aperture $\alpha_{\operatorname{tr}} \approx \gamma z \hat{s}_{*} /\left(\tilde{k} u_{H}\right)$ is shown by the solid vertical line to the right, and the time-reversal resolution $\rho_{\mathrm{tr}} \approx u_{H} / \hat{s}_{*}$ is shown to the far right in the plot.

Appendix A. White-noise limit for the Schrödinger and Wigner equations. In this appendix we state the theorem and the precise conditions under which the convergence to the white-noise models have been rigorously proved in [15] and [13].

First we state the three common assumptions for both cases.

Assumption 1.

$$
\Phi_{(\eta, \rho)}(\overrightarrow{\mathbf{k}}) \leq K\left(\eta^{2}+|\overrightarrow{\mathbf{k}}|^{2}\right)^{-H-1 / 2-d / 2}\left(1+\rho^{-2}|\mathbf{k}|^{2}\right)^{-2}, \quad \overrightarrow{\mathbf{k}} \in \mathbb{R}^{d+1}, \quad H \in(0,1),
$$

for some positive constant $K$. 
Let $\mathcal{F}_{z}$ and $\mathcal{F}_{z}^{+}$be the sigma-algebras generated by $\left\{V_{s}: \forall s \leq z\right\}$ and $\left\{V_{s}\right.$ : $\forall s \geq z\}$, respectively. Let $\mathbb{E}_{z}$ define the expectation conditioned on $\mathcal{F}_{z}$. Define the correlation coefficient

$$
r_{\eta, \rho}(t)=\sup _{\substack{h \in \mathcal{F}_{z} \\ \mathbb{E}[h]=0, \mathbb{E}\left[h^{2}\right]=1}} \sup _{\substack{g \in \mathcal{F}_{z+t}^{+} \\ \mathbb{E}[g]=0, \mathbb{E}\left[g^{2}\right]=1}} \mathbb{E}[h g],
$$

where we emphasize the dependence on the cutoffs $\eta, \rho$ in the subscript.

In the Gaussian case the correlation coefficient $r_{\eta, \rho}(t)$ equals the linear correlation coefficient given by

$$
r_{\eta, \rho}(t)=\sup _{f_{1}, f_{2}} \int e^{i \xi t} f_{1}(\xi, \mathbf{k}) f_{2}(\xi, \mathbf{k}) \Phi_{(\eta, \rho)}(\xi, \mathbf{k}) d \xi d \mathbf{k},
$$

where $f_{1}, f_{2}$ are elements of the Hardy space $\mathcal{H}^{2}$ of $L^{2}\left(\mathbb{R}^{d} ; \Phi_{(\eta, \rho)}\right)$-valued analytic functions in the upper half $\xi$-space satisfying the normalization condition

$$
\int\left|f_{j}(\xi, \mathbf{k})\right|^{2} \Phi_{(\eta, \rho)}(\xi, \mathbf{k}) d \xi d \mathbf{k}=1, \quad j=1,2 .
$$

There are various criteria for the decay rate of the linear correlation coefficients; see [30].

Assumption 2. The correlation coefficient $r_{\eta, \rho}(t)$ is integrable for any $\eta>0$, $\rho<\infty$.

The importance of Assumption 1 lies in the following result.

LEMma A.1.

$$
\tilde{V}_{z}(\mathbf{x})=\int_{z}^{\infty} \mathbb{E}_{z}\left[V_{s}(\mathbf{x})\right] d s
$$

defines a square-integrable $\mathbf{x}$-homogeneous process.

Next we assume the 6th order sub-Gaussian property.

Assumption 3.

$$
\begin{aligned}
\sup _{|\mathbf{y}| \leq L} \mathbb{E}\left[\left[V_{z}^{\varepsilon}\right]^{2}\left[\tilde{V}_{z}^{\varepsilon}\right]^{4}\right](\mathbf{y}) \leq C_{3}\{ & \left(\sup _{|\mathbf{y}| \leq L} \mathbb{E}\left[V_{z}^{\varepsilon}\right]^{2}(\mathbf{y})\right)\left(\sup _{|\mathbf{y}| \leq L} \mathbb{E}^{2}\left[\tilde{V}_{z}^{\varepsilon}\right]^{2}(\mathbf{y})\right) \\
& \left.+\left(\sup _{|\mathbf{y}| \leq L} \mathbb{E}^{2}\left[V_{z}^{\varepsilon} \tilde{V}_{z}^{\varepsilon}\right](\mathbf{y})\right)\left(\sup _{|\mathbf{y}| \leq L} \mathbb{E}\left[\tilde{V}_{z}^{\varepsilon}\right]^{2}(\mathbf{y})\right)\right\}
\end{aligned}
$$

for all $L<\infty$, where the constants $C_{1}, C_{2}$, and $C_{3}$ are independent of $\varepsilon, \eta, \rho, \gamma$.

A.1. The Schrödinger case. In addition, we assume the following. Assumption 4. For any fixed $\eta>0$ and every $\theta \in C_{c}^{\infty}\left(\mathbb{R}^{2}\right)$

$$
\sup _{z<z_{0}}\left\|\theta \tilde{V}\left(\frac{z}{\varepsilon^{2}}, \cdot\right)\right\|_{2}=o\left(\frac{1}{\varepsilon}\right) \quad \forall \varepsilon \leq 1 \leq \rho
$$

with a random constant of finite moments independent of $\rho$ and $\varepsilon$. 
When $V$ is Gaussian, $\tilde{V}$ is also Gaussian, and Assumption 4 is always satisfied:

$$
\sup _{z<z_{0}}\left\|\theta \tilde{V}\left(\frac{z}{\varepsilon^{2}}, \cdot\right)\right\|_{2} \leq \tilde{C} \log \left[\frac{z_{0}}{\varepsilon^{2}}\right]
$$

where the random constant $\tilde{C}$ has a Gaussian-like tail by a simple application of Borell's inequality.

We consider the weak formulation of (3.10),

$$
i \tilde{k}\left[\left\langle\Psi^{\varepsilon}, \theta\right\rangle-\left\langle\Psi_{0}, \theta\right\rangle\right]=-\int_{0}^{z} \frac{\gamma}{2}\left\langle\Psi_{s}^{\varepsilon}, \Delta \theta\right\rangle d s-\frac{\tilde{k}^{2}}{2 \gamma \varepsilon} \int_{0}^{z}\left\langle\Psi_{s}^{\varepsilon}, V\left(\frac{s}{\varepsilon^{2}}, \cdot\right) \cdot \theta\right\rangle d s
$$

for any test function $\theta \in C_{c}^{\infty}\left(\mathbb{R}^{d}\right)$, the space of smooth functions with compact support.

TheOREM A.2. Let $V_{z}^{\varepsilon}$ be a $z$-stationary, $\mathbf{x}$-homogeneous, almost surely locally bounded random process such that Assumptions 1, 2, 3, and 4 are satisfied. Let $\eta>0$ and $\rho<\infty$ be fixed as $\varepsilon \rightarrow 0$. Then the $L^{2}$-weak solution $\Psi^{\varepsilon}$ of (A.9) converges in law to the $L^{2}$-weak solution of the Gaussian white-noise model with the covariance function given by (3.13).

Next we consider the limiting case $\eta=0$. This would induce uncontrollable large scale fluctuation in the Gaussian-Markovian model, which should be factored out first. Thus we consider the solution of the form

$$
\Psi(z, \mathbf{x})=\Psi^{\prime}(z, \mathbf{x}) \exp \left(\frac{i \tilde{k}}{\gamma \sqrt{2}} \int_{0}^{z} \mathcal{B}_{s}(0) d s\right)
$$

and the resulting Schrödinger-Stratonovich equation

$$
d \Psi_{z}=\frac{i}{2 \tilde{k}} \Delta \Psi_{z} d z+\frac{i \tilde{k}}{\sqrt{2} \gamma} \Psi_{z} \circ d \mathcal{B}_{z}^{\prime}, \quad \Psi_{0}(\mathbf{x})=\Psi_{0}(\mathbf{x})
$$

where $\mathcal{B}_{z}^{\prime}$ is given by

$$
\mathcal{B}_{z}^{\prime}(\mathbf{x})=\mathcal{B}_{z}(\mathbf{x})-\mathcal{B}_{z}(0)
$$

with the covariance function

$$
\Gamma^{\prime}(\mathbf{x}, \mathbf{y})=\pi \int\left(e^{i \mathbf{x} \cdot \mathbf{p}}-1\right)\left(e^{-i \mathbf{y} \cdot \mathbf{p}}-1\right) \Phi_{(0, \infty)}(0, \mathbf{p}) d \mathbf{p} .
$$

Note that the above integral is convergent only if

$$
H<1 / 2
$$

in particular, the limit exists for the Kolmogorov value $H=1 / 3$.

A.2. The Wigner case. We consider the weak formulation of the WignerMoyal equation: to find $W_{z}^{\varepsilon} \in D\left([0, \infty) ; L^{2}\left(\mathbb{R}^{2 d}\right)\right)$ such that $\left\|W_{z}^{\varepsilon}\right\|_{2} \leq\left\|W_{0}\right\|_{2} \forall z>0$ and

$$
\left\langle W_{z}^{\varepsilon}, \theta\right\rangle-\left\langle W_{0}, \theta\right\rangle=\tilde{k}^{-1} \int_{0}^{z}\left\langle W_{s}^{\varepsilon}, \mathbf{p} \cdot \nabla_{\mathbf{x}} \theta\right\rangle d s+\frac{\tilde{k}}{2 \varepsilon} \int_{0}^{z}\left\langle W_{s}^{\varepsilon}, \mathcal{L}_{s}^{\varepsilon} \theta\right\rangle d s \quad \forall \theta \in \mathcal{S}
$$

with the test function space $\mathcal{S}$ defined as

$$
\mathcal{S}=\left\{\theta(\mathbf{x}, \mathbf{p}) \in L^{2}\left(\mathbb{R}^{2 d}\right) ; \mathcal{F}_{2}^{-1}[\theta](\mathbf{x}, \mathbf{y}) \in C_{c}^{\infty}\left(\mathbb{R}^{2 d}\right)\right\} .
$$


Here $\mathcal{F}_{2}^{-1}$ stands for the inverse Fourier transform with respect to the second variable (i.e., $\mathbf{p})$.

Assumption 5. For any $\eta>0$,

$$
R_{\eta}=\limsup _{\rho \rightarrow \infty} \int_{0}^{\infty} r_{\eta, \rho}(t) d t<\infty
$$

such that

$$
\limsup _{\eta \rightarrow 0} \eta R_{\eta}<\infty .
$$

For the Gaussian case with the generalized von Kármán spectrum

$$
\Phi_{v k}(\overrightarrow{\mathbf{k}})=2^{H-1} \Gamma\left(H+\frac{d+1}{2}\right) \eta^{2 H} \pi^{-(d+1) / 2}\left(\eta^{2}+|\overrightarrow{\mathbf{k}}|^{2}\right)^{-H-1 / 2-d / 2}, \quad \overrightarrow{\mathbf{k}} \in \mathbb{R}^{d+1},
$$

a straightforward scaling argument shows that

$$
r_{\eta, \rho}(t)=r_{1, \rho}(\eta t),
$$

which is also independent of $\rho$. This motivates Assumption 4.

Define

$$
\begin{aligned}
\delta_{\gamma} V_{z}^{\varepsilon}(\mathbf{x}, \mathbf{y}) & \equiv V_{z}^{\varepsilon}(\mathbf{x}+\gamma \mathbf{y} / 2)-V_{z}^{\varepsilon}(\mathbf{x}-\gamma \mathbf{y} / 2) \\
V_{z}^{\varepsilon}(\mathbf{x}) & =V_{z / \varepsilon^{2}}(\mathbf{x})
\end{aligned}
$$

Assumption 6. For every $\theta \in \mathcal{S}$ and $\tilde{V}_{z}^{\varepsilon}$ given by (A.3), there exists a random constant $C$ such that

$$
\sup _{z<z_{0}}\left\|\delta_{\gamma} \tilde{V}_{z}^{\varepsilon} \mathcal{F}_{2}^{-1} \theta\right\|_{4} \leq \frac{C}{\sqrt{\varepsilon}} \sup _{|\mathbf{x}|,|\mathbf{y}| \leq L} \mathbb{E}^{1 / 2}\left|\delta_{\gamma} \tilde{V}_{z}^{\varepsilon}(\mathbf{x}, \mathbf{y})\right|^{2} \quad \forall \theta \in \mathcal{S}, \varepsilon, \eta, \gamma \leq 1 \leq \rho
$$

with $C$ possessing finite moments and depending only on $\theta, z_{0}$, where $L$ is the radius of the ball containing the support of $\mathcal{F}_{2}^{-1} \theta$.

The same remark following Assumption 3 is applicable here again: Assumption 6 is satisfied by the Gaussian case [13].

TheOREM A.3. Let $V_{z}^{\varepsilon}$ be a z-stationary, $\mathbf{x}$-homogeneous, almost surely locally bounded random process such that Assumptions 1, 2, 3, 5, and 6 are satisfied. Let $\gamma>0$ be fixed.

(i) Let $\eta$ be fixed and $\rho$ be fixed or tend to $\infty$ as $\varepsilon \rightarrow 0$ such that

$$
\lim _{\varepsilon \rightarrow 0} \varepsilon \rho^{2-H}=0 .
$$

Then the weak solution $W^{\varepsilon}$ of the Wigner-Moyal equation with the initial condition $W_{0} \in L^{2}\left(\mathbb{R}^{2 d}\right)$ converges in law to the $L^{2}$-weak solution of the Gaussian white-noise model (3.23) with the covariance operators given by (3.24) with $\eta>0$. The statement holds true for any $H \in(0,1)$.

(ii) Suppose additionally that $H<1 / 2$ and $\eta=\eta(\varepsilon) \rightarrow 0$ such that

$$
\lim _{\varepsilon \rightarrow 0} \varepsilon \eta^{-1}\left(\eta^{-1}+\rho^{2-H}\right)=0 .
$$

Then the same convergence holds true. 


\section{REFERENCES}

[1] F. Bailly, J. P. Clouet, and J. P. Fouque, Parabolic and Gaussian white noise approximation for wave propagation in random media, SIAM J. Appl. Math., 56 (1996), pp. 1445-1470.

[2] G. BAL And L. RYZHIK, Time reversal and refocusing in random media, SIAM J. Appl. Math., 63 (2003), pp. 1475-1498.

[3] M. J. Beran and J. Oz-Vogt, Imaging through turbulence in the atmosphere, Prog. Optics, 33 (1994), pp. 319-388.

[4] J. Berryman, L. Borcea, G. Papanicolaou, and C. Tsogka, Statistically stable ultrasonic imaging in random media, J. Acoust. Soc. Amer., 112 (2002), pp. 1509-1522.

[5] P. Blomgren, G. Papanicolaou, and H. Zhao, Super resolution in time reversal acoustics, J. Acoust. Soc. Amer., 111 (2002), pp. 230-248.

[6] L. Borcea, C. Tsogka, G. Papanicolaou, and J. Berryman, Imaging and time reversal in random media, Inverse Problems, 18 (2002), pp. 1247-1279.

[7] M. Born and E. Wolf, Principles of Optics, Pergamon Press, New York, 1975.

[8] R. S. Cole, K. L. Ho, and N. D. Mavrokoukoulakis, The effect of the outer scale of turbulence and wavelength on scintillation fading at millimeter wavelengths, IEEE Trans. Antennas and Propagation, 26 (1978), pp. 712-715.

[9] F. Dalaudier, A. S. Gurvich, V. Kan, And C. Sidi, Middle stratosphere temperature spectra observed with stellar scintillation and in situ techniques, Adv. Space Res., 14 (1994), pp. 61-64.

[10] D. Dawson and G. Papanicolaou, A random wave process, Appl. Math. Optim., 12 (1984), pp. $97-114$

[11] D. Dowling AND D. JACKSOn, Narrow-band performance of phase-conjugate arrays in dynamic random media, J. Acoust. Soc. Amer., 91 (1992), pp. 3257-3277.

[12] A. Fannjiang, Invariance principle for inertial-scale behavior of scalar fields in Kolmogorovtype turbulence, Phys. D, 179 (2003), pp. 161-182.

[13] A. Fannjiang, White-noise and geometrical optics limits of Wigner-Moyal equation for wave beams in turbulent media, Comm. Math. Phys., to appear.

[14] A. Fannjiang, Self-averaging scaling limits for random parabolic waves, Arch. Ration. Mech. Anal., to appear.

[15] A. FAnnJiang AND K. Sølna, Scaling limits for laser beam propagation in atmospheric turbulence, Stoch. Dyn., 4 (2004), pp. 135-151.

[16] A. Fannjiang And K. Solna, Sound Propagation through a Fractal Layer, preprint, 2003.

[17] R. L. FANTE, Inner-scale size effect on the scintillations of light in the turbulent atmosphere, J. Opt. Soc. Amer., 73 (1983), pp. 277-281.

[18] M. Fink, Time-reversed acoustics, Sci. Amer., 281 (1999), pp. 91-97.

[19] M. FInk AND J. DE Rosny, Time-reversed acoustics in random media and in chaotic cavities, Nonlinearity, 15 (2002), pp. R1-R18.

[20] M. Fink, D. Cassereau, A. Derode, C. Prada, P. Roux, M. Tanter, J. L. Thomas, and F. Wu, Time-reversed acoustics, Rep. Progr. Phys., 63 (2000), pp. 1933-1995.

[21] M. Fink and C. Prada, Acoustic time-reversal mirrors, Inverse Problems, 17 (2001), pp. R1-R38.

[22] J. P. Fouque, G. Papanicolaou, and Y. Samuelides, Forward and Markov approximation: The strong-intensity-fluctuations regime revisited, Waves Random Media, 8 (1998), pp. 303-314.

[23] D. L. Fried, Statistics of a geometric representation of wavefront distortion, J. Opt. Soc. Amer., 55 (1965), pp. 1427-1435.

[24] D. L. Fried, Optical resolution through a randomly inhomogeneous medium for very long and very short exposures, J. Opt. Soc. Amer., 56 (1966), pp. 1372-1379.

[25] D. L. Fried, Limiting resolution looking down through the atmosphere, J. Opt. Soc. Amer., 56 (1966), pp. 1380-1384.

[26] K. Furutsu, Random Media and Boundaries: Unified Theory, Two-Scale Method, and Applications, Springer-Verlag, Berlin, 1993.

[27] P. Gérard, P. Markovich, N. Mauser, and F. Poupaud, Homogenization limits and Wigner transforms, Comm. Pure Appl. Math., 50 (1997), pp. 323-380.

[28] W. Hodgkiss, H. Song, W. Kuperman, T. Akal, C. Ferla, and D. Jackson, A long-range and variable focus phase-conjugation experiment in a shallow water, J. Acoust. Soc. Amer., 105 (1999), pp. 1597-1604.

[29] R. E. Hufnagel and N. R. Stanley, Modulation transfer function associated with image transmission through turbulent media, J. Opt. Soc. Amer., 54 (1964), pp. 52-61. 
[30] I. A. Ibragimov and Y. A. Rozanov, Gaussian Random Processes, Springer-Verlag, New York, 1978.

[31] W. A. Kuperman, W. S. Hodgkiss, H. C. Song, T. Akal, C. Ferla, and D. R. Jackson, Phase conjugation in the ocean: Experimental demonstration of an acoustic time-reversal mirror, J. Acoust. Soc. Amer., 103 (1997), pp. 25-40.

[32] W. Lauterborn and T. Kurz, Coherent Optics: Fundamentals and Applications, SpringerVerlag, Berlin, 2003.

[33] R. M. Manning, Stochastic Electromagnetic Image Propagation and Adaptive Compensation, McGraw-Hill, New York, 1993.

[34] A. S. Monin And A. M. Yaglom, Statistical Fluid Mechanics: Mechanics of Turbulence, Vol. 1, MIT Press, Cambridge, MA, 1971.

[35] G. Papanicolaou, L. RyzhiK, and K. Solna, The parabolic approximation and time reversal, Mat. Contemp., 23 (2002), pp. 139-159.

[36] G. Papanicolaou, L. Ryzhik, and K. Sølna, Statistical stability in time reversal, SIAM J. Appl. Math., 64 (2004), pp. 1133-1155.

[37] G. PaPAnicolaou And K. Solna, Wavelet based estimation of Kolmogorov turbulence, in LongRange Dependence: Theory and Applications, P. Doukhan, G. Oppenmeim, and M. S. Taqqu, eds., Birkhäuser Boston, Boston, MA, 2002, pp. 473-505.

[38] J. W. Strohbehn, Laser Beam Propagation in the Atmosphere, Springer-Verlag, Berlin, 1978.

[39] F. TAPPERT, The parabolic approximation method, in Wave Propagation and Underwater Acoustics, Lecture Notes in Phys. 70, J. B. Keller and J. S. Papadakis, eds., SpringerVerlag, Berlin, 1977, pp. 224-287.

[40] V. I. Tatarski, The Effects of the Turbulent Atmosphere on Wave Propagation, U.S. Department of Commerce, Springfield, VA, 1971.

[41] V. I. Tatarski, A. Ishimaru, and V. U. Zavorotny, eds., Wave Propagation in Random Media (Scintillation), SPIE, Bellingham, WA, IOP Press, New York, 1993.

[42] N. J. Woolf, High resolution imaging from the ground, Annu. Rev. Astron. Astrophys., 20 (1982), pp. 367-398.

[43] C. Y. Young, L. C. Andrews, and A. Ishimaru, Time-of-arrival fluctuations of a space-time Gaussian pulse in weak optical turbulence: An analytic solution, Appl. Opt., 37 (1998), pp. $7655-7660$ 